Sanfter Paternalismus, meritorische

Güter, und der normative Individualismus

Gebhard Kirchgässner

July 2012 Discussion Paper no. 2012-17 


$\begin{array}{ll}\text { Editor: } & \text { Martina Flockerzi } \\ & \text { University of St. Gallen } \\ & \text { School of Economics and Political Science } \\ & \text { Department of Economics } \\ & \text { Varnbüelstrasse 19 } \\ & \text { CH-9000 St. Gallen } \\ & \text { Phone +41 71 224 23 25 } \\ & \text { Fax } \quad+41712243135 \\ & \text { Email seps@unisg.ch } \\ & \text { School of Economics and Political Science } \\ & \text { Department of Economics } \\ & \text { University of St. Gallen } \\ & \text { Varnbüelstrasse 19 } \\ \text { Publisher: } & \text { CH-9000 St. Gallen } \\ & \text { Phone +41 71 224 23 25 } \\ & \text { Fax +41 71 224 31 35 } \\ & \text { http://www.seps.unisg.ch }\end{array}$




\title{
Sanfter Paternalismus, meritorische Güter, und der normative Individualismus ${ }^{1}$
}

\author{
Gebhard Kirchgässner ${ }^{2}$
}

Author's address:

\author{
Prof. Dr. Gebhard Kirchgässner \\ SIAW-HSG \\ Bodanstrasse 8 \\ $\mathrm{CH}-9000$ St. Gallen \\ Phone +41712242340 \\ Fax +41712242298 \\ Email gebhard.kirchgaessner@unisg.ch \\ Website www.siaw.unisg.ch
}

\footnotetext{
${ }^{1}$ Ausführliche schriftliche Fassung eines Vortrags im Rahmen der Tagung ,Grenzen der Konsumentensouveränität“, Evangelische Akademie Tutzing, 8. März 2012. Für Unterstützung danke ich Frau Teresa Körner.

${ }^{2}$ Universität St. Gallen, Schweizerisches Institut für Aussenwirtschaft und Angewandte Wirtschaftsforschung, Leopoldina und CESifo.
} 


\begin{abstract}
Paternalism is an attempt to influence the decisions of individuals for their own benefit even if there are no third parties involved. This seems to be a contradiction to normative individualism which provides the general orientation of our modern democracies. Soft or libertarian paternalism accepts the necessity of paternalism due to the existence of behavioural anomalies, but intends to apply only such measures that do not restrict the decision leeway of individuals. Nevertheless, the same objections which can be raised against its strong version can also be raised against soft paternalism. On the other hand, as soon as we accept that human beings are able to reflect not only on their actions but also on the preferences guiding them, there exists no longer a necessary contradiction between paternalism and normative individualism. As far as we know today, the possibilities to successfully apply soft paternalistic measures are rather limited. On the other hand, while some of its criticisms are justified, others are largely overshooting the mark and seem at least partly to be ideologically motivated.
\end{abstract}

\title{
Keywords
}

Libertarian Paternalism, Soft Paternalism, Merit Goods, Normative Individualism, Democracy.

\section{JEL Classification}

H11, D63, D72. 


\section{$1 \quad$ Einleitung}

[1] Egal ob es um soziale Pflichtversicherungen, um Subventionen für Oper und Theater, um kostenlose Vorsorgeuntersuchungen, um Bereitstellung von Information für Konsumenten durch den Staat, die Anschnallpflicht im Kraftfahrzeug oder die Helmpflicht für Motorradund möglicherweise auch für Fahrradfahrer geht: Paternalismus ist allgegenwärtig. Dabei ist er aus Sicht eines liberalen Weltbilds gegenüber mündigen Bürgerinnen und Bürgern nicht einfach zu rechtfertigen: Er widerspricht - zumindest prima facie - dem Leitbild des normativen Individualismus, welches seit der Aufklärung in unserer abendländischen Kultur dominiert und nach welchem einzig die Individuen die Träger von Werten sind. Staatliche Eingriffe in die individuellen Entscheidungen bedürfen vor diesem Hintergrund besonderer Rechtfertigung. Diese wird typischerweise in der Existenz externer Effekte bzw. der unzureichenden Bereitstellung öffentlicher Güter in Märkten, auf denen (ausschliesslich) private Akteure handeln, gesehen. Entsprechende Eingriffe werden dementsprechend in aller Regel auch nicht dem Paternalismus zugerechnet. Schliesslich werden in diesen Fällen die faktischen Präferenzen der Individuen akzeptiert, auch wenn ihnen nicht unbedingt erlaubt wird, sie auszuleben: Es geht darum, negative Wirkungen auf Dritte zu verhindern, so dass letztlich ein für (fast) alle akzeptables gesellschaftliches Ergebnis herauskommt.

[2] Anders verhält sich dies mit den meritorischen Gütern im Sinne von R.A. MusGrave (1957). Hier geht es nicht um unbeteiligte Dritte, sondern darum, die Menschen so zu beeinflussen, dass sie - zu ihrem eigenen Besten - nicht ihren aktuellen Präferenzen folgen, sondern sich möglichst so verhalten, wie es derjenigen Autorität, welche diese Massnahmen ergreift, vorschwebt. Die von R.A. Musgrave (1957, S. 341) angebotene Begründung für Meritorik ist vor dem Hintergrund eines liberalen Weltbilds kaum akzeptierbar, handelt es sich doch um Eingriffe, die zu Ergebnissen führen sollen, die mit den aktuellen Präferenzen der betroffenen Individuen in Konflikt stehen.

[3] Hinter der liberalen Konzeption steht das Leitbild des mündigen und aufgeklärten Bürgers, der selbst in der Lage ist, seine Interessen zu vertreten, und der seine Entscheidungen im Lichte dieser Interessen und seiner Handlungsmöglichkeiten autonom fällt. Dieses Weltbild liegt auch der traditionellen (bürgerlichen) Ökonomie zugrunde, was nicht weiter verwunderlich ist, ist diese doch in ihren Anfängen ein Kind der Aufklärung und steht bis heute in dieser Tradition. Zumindest als normative Richtschnur individuellen Handelns hat dieses Leitbild auch bis heute kaum etwas an seiner Attraktivität eingebüsst. Anders sieht es freilich aus, wenn man danach fragt, inwieweit die Individuen in ihrem alltäglichen Verhalten diesem Leitbild entsprechen. Hier hat die Verhaltensökonomie in den letzten Jahrzehnten eine ganze Reihe inzwischen gut abgesicherter Erkenntnisse über ,Verhaltensanomalien` aufgezeigt und damit über Verhaltensmuster, die mit diesem Leitbild kaum oder gar nicht vereinbar sind.) Soweit dies jedoch der Fall ist, stellt sich die Frage der Meritorik neu.

[4] Ein Standardbeispiel für eine solche Anomalie bieten die ,401(k)-Pläne` im Rahmen der Altersvorsorge in den Vereinigten Staaten. ${ }^{2)}$ Hier geht es darum, ob die Unternehmen ihre

1. Siehe hierzu z.B. R.H. THALER (1992) sowie G. KIRCHGÄSSNER (2008, S. 202ff.).

2. Siehe hierzu auch B.D. Bernheim, A. Fradkin und I. Popov (2011). - Die Bezeichnung ergibt sich aus der entsprechenden Definition der U.S. Finanzbehörde. Für Einkommensanteile, die in diese Vor- 
Beschäftigten bei der Einstellung automatisch in die betriebliche Altersvorsorge aufnehmen sollen, wobei diese die Möglichkeit haben, dies abzulehnen, oder ob sie ihnen lediglich ein Angebot zur Aufnahme unterbreiten sollen. Bei vollständig rationalen Individuen sollte dies keinen Unterschied machen: In beiden Fällen verfügen sie über die gleiche Information und müssen eine Entscheidung darüber treffen, ob sie diese Versicherung abschliessen wollen. Es geht ausschliesslich darum, ob man auf dem entsprechenden Formular für den Eintritt oder für die Ablehnung ein Kreuz machen muss; es gibt weder bezüglich der Information noch bezüglich der Transaktionskosten irgendeinen Unterschied.

[5] Dennoch gibt es massive Unterschiede in der Teilnahme an solchen Versicherungen. B.C. MADRIAN und D.F. SHEA (2001) zeigen an der Fallstudie eines Unternehmens, dass sich die Teilnahmequote bei Neueinstellungen massiv erhöht, wenn die Beschäftigten automatisch aufgenommen werden. ${ }^{3)}$ Dadurch erhöht sich die Sparquote anfänglich um 50 Prozent. Dieser beträchtliche Effekt ist zwar nicht dauerhaft, da bei nicht-automatischer Aufnahme die Teilnahmequote kontinuierlich ansteigt und nach etwa drei Jahren die mehr oder weniger konstante Quote derer erreicht, die automatisch aufgenommen wurden. Die Differenz in der Ersparnis ist jedoch erheblich und kann daher deutliche Auswirkungen auf die Altersvorsorge haben.

[6] Man kann dies z.B. so interpretieren, dass das, was vom Unternehmen vorgegeben wird, als ,Status Quo‘ betrachtet wird und die Beschäftigten einer Status Quo-Verzerrung unterliegen. ${ }^{4)}$ Man kann es auch als Auswirkung des Framing betrachten. ${ }^{5)}$ Was dieses Beispiel aber auf jeden Fall zeigt, ist, dass bei solchen Entscheidungen die Rückfalloption, d.h. jene Regelung, welche zunächst vorgegeben wird und die dann Gültigkeit erlangt, wenn der Betroffene nicht den Wunsch nach einer anderen Regelung äussert, eine erhebliche Bedeutung hat.

[7] Ein anderes, allgemein bekanntes Faktum ist, dass wir unangenehme Dinge gerne auf morgen ver- bzw. auf die ,lange Bank‘ schieben. Verhielten wir uns vollständig rational, sollten wir dies nicht tun. Zwar macht es Sinn, zukünftige Ereignisse weniger stark zu gewichten als gegenwärtige, d.h. zukünftige Ereignisse in ihrer Bedeutung abzudiskontieren. Dabei sollte die Diskontrate jedoch nicht zu hoch sein: Sie sollte in etwa dem Marktzins entsprechen; er sollte die durchschnittliche Zeitpräferenz der Individuen widerspiegeln. Insbesondere aber sollte sie, wie hoch sie auch immer ist, über den Zeithorizont hinweg konstant sein: Die relative Minderschätzung eines Ereignisses, welches elf Jahre vor uns in der Zukunft liegt, gegenüber einem Ereignis, welches in zehn Jahren geschieht, sollte nicht anders sein als der Vergleich zwischen einem Ereignis in einem Jahr und einem Ereignis heute. Tatsächlich aber

sorgeeinrichtungen eingezahlt werden, müssen zwar Sozialabgaben, aber keine Steuern entrichtet werden. Die Einzahlungen sind freiwillig. Die Steuern werden erst später fällig, wenn daraus Leistungen an die Versicherten erbracht werden. (Die Regelung entspricht insofern derjenigen für die Pensionskassen in der Schweiz.)

3. J.J. CHOI et al. (2003) erhalten ähnliche Ergebnisse in einer Untersuchung für drei andere Unternehmen. Theoretische Begründungen dazu finden sich z.B. in J.J. CHOI et al. (2003a).

4. Zur Status Quo Verzerrung siehe W.F. SAMUELSON und R. ZeCKHAUSER (1988) sowie R.H. THALER (1992, S. 63ff).

5. Zum Framing siehe A. TVERSKY und D. KAHNEMAN $(1981,1987)$ sowie auch V. STOCKÉ (2002). 
machen wir hier massive Unterschiede: Wir verwenden für nahe Ereignisse eine (sehr) hohe Diskontrate, während wir Ereignisse, die weit in der Zukunft liegen, gegeneinander kaum mehr diskontieren. D. LAibson, A. RePETTO und J. ToBACMAn (2007) haben in einer Felduntersuchung über das Sparverhalten amerikanischer Haushalte implizite Diskontraten geschätzt und kamen dabei zum Ergebnis, dass die kurzfristige Rate etwa 40 Prozent beträgt, während die langfristige bei 4.3 Prozent liegt. Während letztere sich damit durchaus in der Nähe des Marktzinses befindet und insofern der traditionellen Theorie entspricht, steht die sehr hohe kurzfristige Rate in starkem Gegensatz zu dieser Theorie.

[8] Inwieweit solche Befunde meritorische Massnahmen rechtfertigen, ist umstritten. Dabei dürfte weitgehend unstrittig sein, dass paternalistische Massnahmen dann, wenn es sich um ,unmündige‘ Personen handelt, angebracht sein können. Dies fängt bei der Erziehung von Kindern an und geht bis zur Behandlung dementer Menschen. Sieht man einmal von Teilen der Bewegung der antiautoritären Erziehung ab, die in den sechziger und siebziger Jahren des vergangenen Jahrhunderts eine Laissez-faire-Pädagogik propagierten, ${ }^{6)}$ dürfte kaum jemand Paternalismus gegenüber (unmündigen) Kindern strikt ablehnen, und auch gegenüber demenzkranken alten Menschen wird man sich (zwangsläufig) paternalistisch verhalten.

[9] Man kann Paternalismus vermutlich als einen allgemeinen Wesenszug des Menschen ansehen; wenn wir es für sinnvoll halten und dazu in der Lage sind, versuchen wir wohl alle, auf die Präferenzen anderer einzuwirken. Dies ist ganz offensichtlich im Rahmen der Sozialisation, aber es wird z.B. auch dann deutlich, wenn wir spenden. Karitative Organisationen machen sich dies zunutze, indem sie konkrete Projekte anbieten. Sie verbinden damit die häufig auch berechtigte - Erwartung, dass dies zu einem höheren Spendenaufkommen führt. Aber auch in ganz alltäglichen Situationen zeigt sich dies. Wir sind eher bereit, einem Bettler einen Teller Suppe zu bezahlen, als ihm den entsprechenden Betrag in Geld zu geben, weil er dieses Geld möglicherweise ,versaufen‘ würde. Dies bedeutet, dass wir einem armen Menschen dann, wenn wir ihm Geld geben, gerne vorschreiben, wie er dieses Geld zu verwenden hat. $^{7)}$

[10] Dass Menschen sich gegenüber anderen paternalistisch verhalten, ist eine Sache, ob dazu staatliche Regulierungen eingesetzt werden sollen, eine andere. Dies kann am Beispiel der Schulpflicht verdeutlicht werden. Dass Eltern ihre Kinder zur Schule schicken, dürfte kaum strittig sein, dass der Staat darauf besteht, dass Kinder zur Schule gehen, schon eher, und dass dies nur staatliche bzw. staatlich lizenzierte Schulen sein dürfen, ist durchaus umstritten. Auch historisch war beides nicht immer miteinander verbunden. So wurde z.B. in Preussen im Jahr 1717 die allgemeine Schulpflicht eingeführt, die aber zunächst eher als Unterrichtspflicht verstanden wurde. Die öffentliche Schule war zwar die Regelschule, aber in bestimmten Ausnahmefällen konnte der Unterricht auch zu Hause durch die Eltern erteilt

6. Zur antiautoritären Erziehung siehe z.B. F. Косн (2000, S. 9ff.).

7. Aus (üblicher) ökonomischer Perspektive ist das tendenziell ineffizient, da dieser Mensch dann, wenn er frei über das Geld verfügen könnte, möglicherweise ein höheres Nutzenniveau erreichen könnte. W.W. Pommerehne (1975) hat jedoch gezeigt, dass eine solche Zweckbindung der Transfers im Sinne des Empfängers sein kann, wenn dadurch die Spendenbereitschaft steigt. 
werden. Einen absoluten Zwang, öffentliche bzw. öffentlich zugelassene Schulen zu besuchen, gibt es in Deutschland erst seit 1938. ${ }^{8)}$

[11] Der Staat verhält sich mit Geboten und Verboten jedoch nicht nur gegenüber Kindern bzw. unmündigen Menschen paternalistisch, sondern auch gegenüber mündigen Erwachsenen, indem er ihnen z.B. eine bestimmte Art der Altersvorsorge vorschreibt oder indem er ihnen den Handel oder sogar den Konsum bestimmter Rauschmittel verbietet (und gleichzeitig den anderer zulässt). Er verhindert damit in bestimmten Bereichen bewusst, dass sich die Individuen frei entscheiden können.

[12] Damit stellt sich die Frage, inwieweit solche Eingriffe gerechtfertigt werden können. Die Tatsache ihrer Existenz rechtfertigt sie nicht, andererseits schafft sie die Tatsache, dass sie mit einem bestimmten Menschenbild nur schwer vereinbar sind, auch nicht aus der Welt, und dies bedeutet auch nicht, dass sie nicht doch gerechtfertigt sein könnten. Dies soll im Folgenden erörtert werden, wobei wir zunächst noch näher auf den Paternalismus eingehen (Abschnitt 2), bevor wir im 3. Abschnitt eine mögliche Begründung der Meritorik vorstellen. Abschnitt 4 befasst sich mit möglichen Anwendungsbereichen des sanften Paternalismus, während im 5. Abschnitt Probleme und Einwände behandelt werden. Die Arbeit schliesst mit einer thesenartigen Zusammenfassung (Abschnitt 6). ${ }^{9}$

\section{Staatlicher Paternalismus vs. normativer Individualismus}

[13] Betrachtet man die Gesamtheit paternalistischer Massnahmen des Staates, so kann man drei Varianten unterscheiden:

(i) den starken (bzw. harten) Paternalismus; hier setzt der Staat Ge- und Verbote ein. Dies betrifft z.B. Pflichtversicherungen oder das Verbot des Handels und des Konsums bestimmter Drogen.

(ii) den weichen Paternalismus; hier verwendet der Staat Steuern und Subventionen. Beispiele sind die Tabaksteuer oder Subventionen für Kulturbetriebe.

(iii) den sanften (bzw. libertären) Paternalismus; hier geht es ausschliesslich um die Bereitstellung und Aufbereitung von Information. Das klassische Beispiel hierzu sind die oben angesprochenen 401(k)-Pläne im System der Altersvorsorge der Vereinigten Staaten. ${ }^{10)}$

Während somit der harte Paternalismus bestimmtes Handeln vollständig unterbinden will, erschwert es der weiche Paternalismus ,nur`, während der sanfte Paternalismus ausschliess-

8. Andere Länder, wie z.B. die Vereinigten Staaten, kennen keinen solchen Zwang. Dort wird die Schulpflicht nach wie vor als Unterrichtspflicht verstanden. Zur Geschichte der Schulpflicht in Deutschland siehe z.B. A. Mors (1986).

9. Siehe hierzu auch meine früheren Ausführungen zu diesem Thema in G. KIRCHGÄsSNER (2008, S. 261ff.).

10. Zur Einführung in den sanften Paternalismus siehe z.B. G. LOEWENSTEIN und E. HAISLEY (2008), die darin eine neue normative Ökonomik sehen. Der Begriff , libertärer Paternalismus' stammt von C.R. SUNSTEIN und R.H. ThALER (2003). Siehe hierzu auch R.H. THALER und C.R. SunsteIN (2003). - Eine andere Unterscheidung zwischen hartem und weichem Paternalismus unter Bezug auf J. FEINBERG (1986) findet sich z.B. bei T.M. Pope (2004), der diesen harten Paternalismus auch verteidigt. Zur Diskussion der Position von J. FEINBERG siehe auch R.J. ARNESON (2005). 
lich über eine Beeinflussung der Informationsaufnahme wirken will. Dass in bestimmten (vielleicht auch vielen) Fällen die Übergänge fliessend sind, ist trivial, spielt jedoch für unsere Argumentation hier keine Rolle. Zentral aber ist für alle Arten des Paternalismus, dass es um die Beeinflussung der Individuen zu ihrem eigenen Wohl und nicht um die Berücksichtigung der Rechte und Interessen Dritter geht.

[14] Im Gegensatz zu den härteren Versionen will der sanften Paternalismus die Möglichkeit der eigenen Entscheidung offen halten. Man geht davon aus, dass man zwischen den ,wahren“ (langfristigen) und den ,geäusserten` (kurzfristigen) Präferenzen unterscheiden kann, und dass die geäusserten Präferenzen durch Verhaltensanomalien verzerrt sein können. Man respektiert insofern die wahren, aber nicht notwendigerweise auch die geäusserten Präferenzen der Individuen. Die Differenz zwischen den beiden Arten von Präferenzen versucht man zum einen durch Ausgleich von Informationsasymmetrien zu überwinden und zum anderen durch ,weiche' Hinweise bzw. Anstösse (,Nudges'), nicht aber durch harte Anreize wie Steuern oder Ge- und Verbote. ${ }^{11)}$ Dabei sollen die Anreize asymmetrisch wirken: Sie sollten so beschaffen sein, dass sie denjenigen helfen, die von Entscheidungsanomalien betroffen sind, aber jene nicht oder nur ganz gering belasten, die vollständig rational sind. ${ }^{12)}$ Solche Verzerrungen zwischen geäusserten und wahren Präferenzen können auch durch gesetzliche Regelungen gemildert oder gar beseitigt werden. ${ }^{13)}$

[15] Zum sanften Paternalismus gehört auch die Zurverfügungstellung von Information, auch wenn dies z.B. bei den von R.H. ThALER und C.R. Sunstein (2008) vorgeschlagenen Massnahmen keine sehr zentrale Rolle spielt. Auch hier geht es darum, die Entscheidungen der Individuen zu verbessern, indem z.B. Informationsasymmetrien ausgeglichen werden, und auch hier spielen bei der Begründung dieser Massnahmen keine Auswirkungen auf Dritte eine Rolle. $^{14)}$

[16] Unabhängig davon, ob es sich um harten oder sanften Paternalismus handelt, bleibt das grundsätzliche Problem der Begründung solcher Handlungen bzw. Massnahmen, auch wenn es sich in unterschiedlicher Schärfe stellt. Es besteht - zumindest prima facie - ein Konflikt mit dem Leitbild des normativen Individualismus. Nun kann man einen Teil dieser Massnahmen mit externen Effekten und/oder Verteilungsargumenten rechtfertigen. Dies gilt z.B. für die Haftpflicht-Pflichtversicherung für Kraftfahrzeuge. Damit soll sichergestellt werden, dass bei einem Unfall die erforderlichen Schadensersatzzahlungen auch geleistet werden können. Ähnlich kann man für eine minimale Pflichtversicherung in der Altersvorsorge argumentieren. Hier soll verhindert werden, dass einzelne Bürgerinnen und Bürger im Alter der Allgemeinheit zur Last fallen, weil sie dann, wenn sie kein Arbeitseinkommen mehr haben und auch nicht für eine Rente vorgesorgt haben, Sozialhilfe aus Steuermitteln beziehen müssen. ${ }^{15}$

11. Siehe hierzu insbesondere R.H. THALER und C.R. SUNSTEIN (2008).

12. Siehe hierzu C. CAMERER et al. (2003, S. 1212). Die Autoren sprechen in diesem Zusammenhang auch von ,Asymmetrischen Paternalismus‘.

13. Siehe hierzu auch CH. Jolls und C.R. SunsteIN (2006).

14. Insofern liegt H. BЕСK (2010, S. 50f.) falsch, wenn er die Behebung von Informationsasymmetrien durch staatliche Informationspolitik nicht zum Paternalismus rechnen will.

15. In diesem Sinne deckt z.B. die erste Säule der schweizerischen Altersvorsorge, die Alters- und Hinterlassenenversicherung (AHV), nur in etwa das Existenzminimum ab. (Dies gilt freilich nur für die Ma- 
Die gleiche Argumentation gilt jedoch nicht mehr, wenn die staatlich vorgeschriebene Altersvorsorge über das Existenzminimum hinausgeht. Mündige Bürger, so lautet das Gegenargument, sollten selbst die Freiheit haben, darüber zu entscheiden, wie und wann sie ihr Lebenseinkommen verwenden, solange daraus keine negativen Effekte für unbeteiligte Dritte entstehen. Konsequenterweise wird von bestimmten politischen Kreisen auch die Abschaffung staatlicher Vorschriften bezüglich der Altersvorsorge gefordert, soweit diese über die Sicherung des Existenzminimums hinausgeht. ${ }^{16)}$

[17] Der paternalistische Staat arbeitet jedoch nicht nur mit Ge- und Verboten, sondern auch, indem er die relativen Preise verändert, und zwar auch dort, wo dadurch keine externen Kosten oder Nutzen ausgeglichen werden. So gibt es z.B. neben dem Verbot des Verkaufs alkoholischer Getränke an Jugendliche besondere Steuern auf diese Getränke, die auch das Verhalten Erwachsener beeinflussen sollen. Einen Teil solcher Steuern kann man mit externen Kosten rechtfertigen, so z.B. die Zigarettensteuer mit jenen Kosten, die der Gesellschaft durch Krankheiten entstehen, welche durch das Rauchen verursacht werden. Und die Mineralölsteuer wird in aller Regel mit den externen Kosten des Verkehrs begründet, wobei die Auffassungen darüber, welcher Unter- oder Überdeckungsgrad durch die heutige Höhe dieser Steuern tatsächlich erreicht wird, weit auseinander gehen. ${ }^{17)}$ Auch die Subvention von Impfungen kann man auf solche Weise rechtfertigen, da dann, wenn sich ein erheblicher Teil der Bevölkerung impfen lässt, wegen des dadurch verminderten Risikos der Ausbreitung der entsprechenden Krankheit auch der Rest der Bevölkerung geschützt wird.

[18] Die Subvention von Opernhäusern oder Theatern, die heute deutlich über 80 Prozent der gesamten Aufwendungen betragen können, lässt sich mit solchen Argumenten jedoch nicht mehr rechtfertigen. ${ }^{18)}$ Man kann sie als Rent-Seeking der Mittelschicht (bzw. genauer eines bestimmten Teils der Mittelschicht, der sich als kulturelle Elite versteht) auf Kosten der Oberschicht und derjenigen Mitglieder der Unterschicht verstehen, die Steuern zahlen. Die Mitglieder der Oberschicht könnten sich auch nicht subventionierte Eintrittskarten leisten; sie zahlen an Steuern vermutlich erheblich mehr, als sie durch die Subventionierung gewinnen. Angehörige der Unterschicht gehen andererseits kaum ins Theater und noch weniger in die Oper, tragen aber mit ihren Steuern die Kosten mit. ${ }^{19)}$ Dagegen gewinnen die Angehörigen der Mittelschicht, die die grösste Nachfrage nach diesen Leistungen entfalten, da sie durch die Verbilligung der Eintrittskarten mehr gewinnen als sie durch die zusätzlichen Steuern verlieren. Sie gehen dann auch häufiger in die Oper oder ins Theater, als wenn es diese Subventio-

ximalrente; erhält jemand weniger Rente und hat er kein zusätzliches Einkommen oder Vermögen, hat er, um das Existenzminimum zu sichern, Anspruch auf Ergänzungsleistungen.)

16. Siehe hierzu z.B. D. DE Pury, H. Hauser und B. Schmid (1995, S. 62ff.).

17. Zur Berechnung der externen Kosten des Verkehrs siehe z.B. E. QUinet (2004).

18. Die eigenen Einnahmen der 144 Theaterunternehmen in Deutschland (einschliesslich Musiktheater) betrugen in der Spielzeit 2008/2009 gerade einmal 17 Prozent der gesamten Ausgaben. Im Durchschnitt wurde jeder Eintritt mit $99.31 €$ subventioniert. Quelle der Daten: Statistisches Jahrbuch 2011 für die Bundesrepublik Deutschland, Tabelle 7.6, S. 180.

19. Jene Mitglieder der Unterschicht, die aufgrund ihres geringen Einkommens nicht steuerpflichtig sind, werden dadurch nicht direkt betroffen. Sie werden aber möglicherweise indirekt betroffen, falls der Staat zur Finanzierung dieser Subventionen Leistungen kürzt, die ihnen zugutegekommen wären. 
nen nicht gäbe. ${ }^{20)}$ Selbstverständlich gibt es Versuche, diese Subventionen zu rechtfertigen, aber sie sind nicht unbedingt überzeugend. ${ }^{21)}$ Zumindest kann man die dadurch bewirkte Umverteilung zugunsten der Mittelschicht damit kaum rechtfertigen.

[19] R.A. Musgrave (1957, S. 341) hat diese Problematik aufgegriffen und für jene Güter, deren Konsum der Staat dadurch zu beeinflussen sucht, dass er die relativen Preise beeinflusst, obwohl keine externen Effekte vorliegen, den Begriff ,meritorische Bedürfnisse“ eingeführt. (Heute spricht man eher von meritorischen Gütern.) Er beschreibt dieses Phänomen zwar, aber findet keine überzeugende Begründung für diese Staatseingriffe. Dies gilt, zumindest solange man von der liberalen Grundüberzeugung ausgeht, dass die Bürgerinnen und Bürger selbst am besten wissen, was für sie gut ist und was nicht, und dass auch der demokratisch legitimierte Staat nicht befugt ist, sich darüber Urteile anzumassen.

[20] Konsequenterweise haben liberale Denker sich schon früh gegen solche Eingriffe gewandt. Zwar fragt z.B. John STUART MiLL in „On Liberty“ (eher rhetorisch):

„Wenn anerkanntermassen Kindern und Minderjährigen Schutz gegen ihre eigene Unreife gebührt - ist die Gesellschaft nicht ebenso verpflichtet, ihn Personen in reifem Alter zu leisten, die genauso wenig zur Selbstbeherrschung fähig sind? Wenn die Spielwut, die Trunksucht, die Unmässigkeit, Müssiggängerei und Unreinlichkeit ebenso der menschlichen Wohlfahrt schädlich und dem Fortschritt hinderlich sind wie viele oder die meisten der gesetzlich verbotenen Handlungen - warum, so könnte gefragt werden, sollte nicht das Gesetz, soweit das angemessen erscheint, auch deren Unterdrückung sich zum Ziel setzen?" (1859, S. 219),

aber er bleibt dabei, dass hier staatliche Eingriffe nur dann gerechtfertigt sein können, wenn Interessen Dritter im Spiel sind:

„Sobald irgendein Teil der Handlungsweise eines Menschen die Interessen anderer nachteilig berührt, erhebt die Gesellschaft den grundsätzlichen Anspruch, über ihn zu richten. Ganz anders aber liegt der Fall, wenn die Handlungsweise eines Menschen nur seine eigenen Interessen berührt oder zwar die Interessen anderer, aber mit deren Zustimmung (wobei vorausgesetzt wird, dass diese Personen grossjährig sind und den normalen Menschenverstand besitzen). In diesen Fällen sollte völlige Freiheit herrschen, gesetzliche wie gesellschaftliche, die Tat zu unternehmen und ihre Folgen zu tragen" (1859, S. 213).

[21] Dieser Tradition folgend wird Paternalismus von Ökonomen häufig abgelehnt. Dies gilt für jeden und damit auch für den sanften Paternalismus. Die Begründung dafür läuft üblicherweise auf die Betonung der Unvereinbarkeit mit dem klassischen liberalen Weltbild (bzw. mit dem traditionellen ökonomischen Ansatz) hinaus. ${ }^{22)}$ Interessanterweise sind Philosophen und Juristen dem Paternalismus gegenüber sehr viel aufgeschlossener, aber nicht deshalb,

20. Zu einer solchen Argumentation siehe z.B. G.A. WITHERS (1979).

21. Zu möglichen Begründungen für die öffentliche Subventionierung der Kunst siehe z.B. B.S. FREY und W.W. POMMEREHNE (1990).

22. Siehe hierzu z.B. Th.C. LeOnard (2008), im deutschen Sprachraum z.B. M. TIETZEL und Ch. MÜLler (1998) sowie J. SCHNELLENBACH $(2011,2012)$. 
weil sie das liberale Weltbild nicht teilen würden, sondern weil sie die Menschen auch als moralische Wesen ansehen, die gegen andere wie auch gegen sich selbst moralische Verpflichtungen haben. ${ }^{23)}$

[22] Dazu kommt, dass man fragen kann, ob ein konsequenter Anti-Paternalismus, wie er von einigen Ökonomen vertreten wird, überhaupt sinnvoll ist. Ergebnisse des Paternalismus sind z.B. auch Arbeitsschutzgesetze: Sie sollen die Menschen in ihrem eigenen Interesse in einem Masse zur Vorsicht zwingen, wie sie dies sonst unter Umständen nicht täten. Man kann dies z.B. mit asymmetrischer Information, aber auch mit unterschiedlichen Vermeidungskosten rechtfertigen: Die einzelnen Arbeitnehmer unterschätzen möglicherweise die Gefahren, und der Arbeitgeber kann die erforderlichen Vorsichtsmassnahmen mit geringeren Kosten durchführen als die einzelnen Arbeitnehmer. Dies ist einer jener Fälle, wo staatliche Eingriffe zu höherer Effizienz führen können, ohne dass man hier externe Effekte bemühen müsste.

[23] Daneben stellt sich die Frage, ob ein strikter Anti-Paternalismus überhaupt durchzuhalten wäre. In letzter Konsequenz würde er nicht nur die Freigabe jeglicher Drogen (zumindest für Erwachsene) und die Abschaffung einer Pflicht-Krankenversicherung bedeuten, sondern auch die Zulassung von Polygamie und Sklaverei, soweit dies alles auf freiwilliger Basis geschieht. Auch ein Straftatbestand ,unterlassene Hilfeleistung' ist mit einem strikten AntiPaternalismus kaum vereinbar. Es fragt sich sehr, ob dies eine Welt wäre, in der wir zu leben wünschten. Die Chance auf eine Durchsetzung dieser Doktrin ist freilich in der Demokratie sehr gering. Wie die Schweiz mit ihrer direkten Demokratie zeigt, stimmen Mehrheiten der Stimmbürgerinnen und Stimmbürger nicht nur für (paternalistische) Sozialversicherungen, sondern auch für die Subvention staatlicher Theater und Opernhäuser.

\section{Zur möglichen Rechtfertigung der Meritorik}

[24] Auch wenn der Paternalismus allgegenwärtig ist und eine Gesellschaft ohne jeglichen Paternalismus kaum erstrebenswert sein dürfte, ist noch nicht geklärt, wie Paternalismus gerechtfertigt werden kann, solange man auf der Grundlage des normativen Individualismus argumentiert. Tatsächlich dürfte es dann, wenn man wie im traditionellen ökonomischen Modell von einer Nutzenfunktion ausgeht, die zwar viele Dimensionen, aber nur eine Ebene kennt, kaum möglich sein, eine konsistente Rechtfertigung für Meritorik zu finden. Hier versagt der Versuch von R.A. Musgrave (1957). ${ }^{24)}$ Aber auch andere Phänomene sind mit diesem Ansatz nicht vereinbar, insbesondere jene der Willensschwäche und der Zeitinkonsistenz. Hier versagt das traditionelle ökonomische Modell.

[25] Anders sieht dies aus, wenn man davon ausgeht, dass die Menschen nicht einfach (in sich konsistente) Präferenzordnungen besitzen, sondern dass sie auch ihr eigenes Wollen und Handeln beurteilen können, dass sie - technisch gesprochen - hierarchisch geordnete Präferenzen haben. Diese für die ökonomische Tradition eher neue und ungewöhnliche Überlegung

23. Siehe z.B. G. Dworkin (2005), der von „moralischem Paternalismus“ spricht, aber auch TH.M. POPE (2004, 2005, 2005a), R. Shafer-LANDAu (2005), P. D. MARnEFFE (2006), J.C. SACHA (2007), oder W. GLOD (2008).

24. Zur früheren Diskussion der Meritorik siehe auch J.G. HEAD (1966), N. ANDEL (1984) sowie K. SCHMIDT (1988). 
kann in der philosophischen Tradition bis auf ARISTOTELES zurückgeführt werden, der in seiner Nikomachischen Ethik von einer „Scheidung ... zwischen dem rationalen Seelenteil und dem irrationalen“ spricht, wobei es „eine wechselseitige Gerechtigkeit auch für diese Teile gebe, wie sie zwischen Herrscher und Beherrschtem besteht. ““25) Und H.G. FRANKFURT (1971) argumentiert, dass die Existenz übergeordneter (moralischer) Präferenzen, mit denen man seine eigenen untergeordneten, aktuellen Präferenzen beurteilen kann, eine der Vorbedingungen dafür ist, dass man Menschen als ,Personen“ mit freiem Willen betrachten kann, da es dafür notwendig ist, dass sie sich ihres Willens bewusst sind und über ihn reflektieren können. $^{26)}$

[26] Dieses Modell hierarchisch geordneter Präferenzfunktionen, welches z.B. von R.H. THALER und H.M. SHEFRIN (1981) mit ihrem Modell vom ,Planer` und ,Doer` auf ökonomische Fragestellungen angewendet wurde, erlaubt es, Selbstbindungen zu erklären, mit denen Individuen dem Problem der Wissensschwäche begegnen. ${ }^{27)}$ Selbstbindungen können freilich nicht nur individuell erfolgen, sondern auch mit Hilfe kollektiver Mechanismen, d.h. die Meritorik kann als eine Art kollektiver Selbstbindung interpretiert werden. Wenn wir z.B. wissen, dass wir in der Gefahr sind, zu wenig für das Alter vorzusorgen, können wir uns über den politischen Prozess und damit kollektiv selbst binden, indem wir dafür sorgen, dass bestimmte Vorschriften erlassen werden, denen wir uns dann wohl oder übel unterordnen müssen. Die übergeordneten langfristigen Präferenzen veranlassen uns, über den politischen Prozess ein Zwangssparen einzuführen, da wir wissen, dass wir aufgrund unserer untergeordneten kurzfristigen Präferenzen sonst kaum jemals die erforderlichen Ersparnisse beiseitelegen würden.

[27] Die Möglichkeit kollektiver Selbstbindung ist die Begründung, die G. BRENNAN und L. LOMASKY (1985) für die Existenz meritorischer Güter geben. Sie sprechen von den übergeordneten als ,reflektive‘ Präferenzen, und sie zeigen, dass sich diese über den politischen Prozess eher als über den Markt durchsetzen, da bei Wahlen und Abstimmungen für die Individuen die Kosten, die sich für sie selbst aus ihrer individuellen Entscheidung ergeben, im Allgemeinen sehr gering und damit vernachlässigbar sind: Die Individuen handeln, wie $\mathrm{H}$. KLIEMT (1986) es formuliert hat, hinter einem „Schleider der Insignifikanz“, ${ }^{28)}$ weshalb man hier eher den reflektiven Präferenzen folgen kann als im Markt, wo die Kosten von (Fehl-) Entscheidungen sofort anfallen. Dies ist die bisher wohl überzeugendste Begründung für meritorische Eingriffe des Staates. G. BRENNAN und L. LOMASKY (1985) weisen auch darauf hin, dass dieses Konzept mit dem normativen Individualismus verträglich ist. Damit ist es aber

25. ARISTOTELES, Nikomachische Ethik, 1138b, S. 121. - Zu einer kurzen Darstellung der philosophischen Tradition siehe G. BRENNAN und L. LOMASKY (1985, S. 195f.).

26. A.K. SEN (1977) argumentiert ähnlich mit einer Hierarchie von Präferenzen, die sogar mehr als zwei Ebenen umfasst.

27. Siehe hierzu auch den klassischen Beitrag von J. ElSTER (1979) sowie J. ELSTER (2000) und T.C. SCHELLING $(1978,1980)$.

28. Siehe hierzu auch G. KIRCHGÄSSNER (1992, 2010). 
auch nicht mehr möglich, diesen als grundsätzlichen Einwand gegen die Meritorik zu verwenden. $^{29)}$

[28] Selbstverständlich können damit nicht alle heute erfolgenden derartigen Eingriffe gerechtfertigt werden. Es dürfte vermutlich nicht einfach sein, die Anschnallpflicht im Kraftfahrzeug oder die Helmpflicht bei Motorrad- und Fahrradfahrern als Ergebnisse kollektiver Selbstbindung zu interpretieren. Auch die Argumentation, dadurch Kostenverlagerungen auf Dritte verhindern zu wollen, die dadurch entstehen, dass die Kosten von Unfällen ohne Gurt bzw. Helm deutlich höher ausfallen und dann von allen Versicherten getragen werden müssen, überzeugt kaum; schliesslich kann man dies auch dadurch erreichen, dass man für diejenigen, die ohne Gurt bzw. Helm fahren (wollen), die Versicherungsleistungen beschränkt bzw. von ihnen höhere Prämien verlangt. Damit wird es schwer, diese Pflichten mit dem Leitbild des normativen Individualismus in Einklang zu beringen. Dies bedeutet freilich nicht notwendigerweise, dass über die Einführung solcher Pflichten nicht im demokratischen Prozess entschieden werden dürfte.

[29] Werden solche Regeln im demokratischen Prozess bestimmt, liegt zunächst auch kein Eingriff in die Souveränität der Konsumenten vor: schliesslich stimmen wir ihm freiwillig zu. Ein solcher Eingriff liegt nur insofern vor, als sich auch die überstimmte Minderheit an diese Regelung halten muss. Dies ist freilich eine Eigenschaft jedes demokratischen Prozesses, soweit nicht Einstimmigkeit gefordert ist, was freilich nur in sehr kleinen Gruppen praktikabel ist. $^{30)}$ Der Eingriff sollte daher, so die Forderung des sanften Paternalismus, so schonend als möglich sein. ${ }^{31)}$

[30] Analog zum Modell hierarchisch geordneter Präferenzordnungen ist das Modell der Verfassungsökonomik, wie wir es von J.M. BuCHANAN und G. TULLOCK (1962) kennen, wie es aber auch in J. RAWLS (1971) verwendet wird. Dort werden zwei Ebenen unterschieden: die Verfassungsebene (die Ebene des Urzustands) und die Ebene des laufenden politischen Prozesses. Auf der ersten Ebene werden die Entscheidungen hinter einem Schleier der Ungewissheit getroffen, d.h. die Individuen wissen nicht, wie sie später - im laufenden politischen Prozess - von den Regeln, über die sie jetzt entscheiden sollen, genau betroffen sein werden. Daher sind hier langfristige Überlegungen bzw. die reflektiven Präferenzen relevant, während später die aktuellen Präferenzen massgebend sind. ${ }^{32)}$ Auf der Verfassungsebene werden die Regeln festgelegt, nach denen später entschieden wird. Analoges geschieht im Rahmen der kollektiven Selbstbindung, wenn man davon ausgeht, dass - angesichts der eingeschränkten Information - auch hier die reflektiven Präferenzen dominieren.

29. T. GRÜNE-YANOFF (2012) bestreitet dies und sieht auch den Libertären Paternalismus in Konflikt mit liberalen Prinzipien, aber er nimmt den Ansatz von G. BRENNAN und L. LOMASKY (1985) nicht zur Kenntnis.

30. Selbst K. WiCKSELL (1896), der für solche Abstimmungen im Prinzip Einstimmigkeit fordert, gestand zu: „auf absolute Einstimmigkeit wird wohl schon aus praktischen Gründen verzichtet werden müssen“ (S. 116).

31. Siehe hierzu z.B. A. v. AAKEN (2006).

32. Siehe hierzu auch J.M. BuCHANAN (1982). 


\section{$4 \quad$ Anwendungsbereiche des sanften Paternalismus}

[31] Es gibt eine ganze Reihe von Vorschlägen, wie Anstösse zu vernünftigerem Verhalten gegeben werden könnten. ${ }^{33)}$ Dabei geht es im Wesentlichen um drei Anomalien, die überwunden bzw. ausgenutzt werden sollen: die Status Quo-Verzerrung, die Willensschwäche und die Zeitinkonsistenz, wobei die letzten beiden häufig eng zusammenhängen. Die wichtigsten Anwendungsbereiche sind die Altersvorsorge und das Gesundheitswesen, wobei bei letzterem auch die Frage der Organspende thematisiert wird.

[32] Im Bereich der Altersvorsorge geht es zum einen um die Ausnützung des Status QuoBias im Rahmen der oben diskutierten 401(k)-Sparpläne. Ein anderer Vorschlag, der auf die Überwindung der Zeitinkonsistenz zielt, geht auf eine Untersuchung von R.H. THALER und S. BENARTZI (2004) zurück. Der hohen Rate der Diskontierung der nahen Zukunft kann dadurch entgegengewirkt werden, dass man den Individuen Sparverträge anbietet, die erst in Zukunft wirksam werden. Dies ist z.B. dann der Fall, wenn vereinbart wird, dass von zukünftigen Einkommenssteigerungen solange ein grösserer Teil gespart wird, bis eine bestimmte, im Vorhinein festgelegte Sparquote erreicht ist. Die Autoren zeigen in einer Fallstudie, die drei Firmen umfasst, dass sich die Sparquote der beteiligten Arbeitnehmer innerhalb von 40 Monaten fast vervierfachte, von 3.5 auf 13.6 Prozent ihres Einkommens. Dies geschah, obwohl sie jederzeit die Möglichkeit hatten, sich aus diesem Programm ohne Nachteile zu verabschieden. Da die Beteiligung an diesem Programm absolut freiwillig war und dennoch das Ziel einer Erhöhung der Sparquote erreicht wurde, bezeichnen R.H. THALER und S. BENARTZI (2004) dieses Programm auch als ein Beispiel für libertären Paternalismus.

[33] Im Gesundheitsbereich werden neben anderem die ,Sündensteuern' thematisiert, d.h. Steuern auf den Genuss von Alkohol, Nikotin und fettem Essen. Dabei wird zunächst diskutiert, inwieweit sie überhaupt gerechtfertigt werden können. (Die oben angesprochene Möglichkeit, dass diese Steuern für negative externe Effekte, die durch diesen Konsum hervorgerufen werden, einen Ausgleich bilden, wird dabei nicht in Betracht gezogen.) Nach der oben wiedergegebenen Argumentation von JOHN STUART MiLl sind solche Eingriffe nicht gerechtfertigt. Zudem sind sie regressiv, da die Bezieher niedriger Einkommen - ceteris paribus - relativ mehr Geld für Zigaretten (bzw. Alkohol oder fettes Essen) ausgeben. Sie machen dadurch die Einkommensverteilung noch ungleichmässiger. Dem entgegnen J. GRUBER und B. Koszegi (2002), dass solche Steuern möglicherweise sogar progressiv sein können, wenn das Steueraufkommen zur Verringerung anderer Steuern verwendet wird, da die Bezieher niedriger Einkommen stärker auf die Preisveränderung der Zigaretten reagieren. Und die Nichtraucher aller Einkommensschichten stellen sich auf jeden Fall besser.

[34] T. O'Donoghue und M. RABIn (2003) schlagen als Alternative zur heutigen Besteuerung ein Modell vor, in dem der Kauf von Zigaretten verboten ist, es sei denn, man hat vorher eine Lizenz z.B. zum Preis von 5000 U.S. Dollar erworben, um 2500 Packungen Zigaretten kaufen zu dürfen. Die Zigaretten selbst wären dann steuerfrei. Süchtige Raucher würden in diesem Modell nicht schlechter fahren als im heutigen System, aber die Schwelle, mit dem Rauchen zu beginnen, läge wesentlich höher und würde vermutlich viele potentielle Einstei-

33. Siehe z.B., die Vorschläge in R.H. THALER und C.R. Sunstein (2008), speziell für die Niederlande auch P. Korman und H. PRAST (2010). 
ger abschrecken. Geht man davon aus, dass zumindest einige von ihnen entsprechend ihren langfristigen Präferenzen nicht rauchen wollen, wäre dieses System gegenüber der heutigen Situation eine Pareto-Verbesserung, d.h. niemand würde schlechter, aber zumindest einige Individuen würden besser gestellt. ${ }^{34)}$

[35] Es ist offensichtlich, dass dieser Vorschlag an praktischen Schwierigkeiten scheitern dürfte. Schliesslich müsste man jeglichen Zwischenhandel unterbinden, indem man ihn z.B. unter Strafe stellt und/oder sicherstellt, dass nur noch diejenigen, die eine solche Lizenz persönlich erworben haben, Zigaretten erhalten. Abgesehen davon, dass dies einen neuen Schwarzmarkt eröffnen würde, könnte man angesichts der jetzt notwendigen Kontrollen hier nicht mehr von einem sanften oder libertären Paternalismus reden. Dennoch macht dieses Modell eine wesentliche Intention der Vertreter dieses Konzepts deutlich: Nach Möglichkeit soll niemand durch die ergriffenen Massnahmen schlechter gestellt werden. ${ }^{35)}$

[36] Übergewicht wird zunehmend in Europa aber insbesondere in den Vereinigten Staaten zum Problem. ${ }^{36)}$ Eine praktikable Möglichkeit, den Anteil der Bevölkerung zu verringern, der extremes Übergewicht hat und der in den Vereinigten Staaten in den vergangenen Jahrzehnten sehr stark zugenommen hat, ist, im Supermarkt besonders fetthaltige Lebensmittel so auszulegen, dass sie vergleichsweise schwer erreichbar sind, und wenig kalorienhaltige Lebensmittel so, dass sie einfach erreichbar sind. Dis gilt insbesondere für jene Lebensmittel, die für Kinder besonders attraktiv sind. Dass die Anordnung der Waren in einem Geschäft bzw. Supermarkt einen Einfluss darauf hat, wie viel davon gekauft wird, ist aus der Marketingliteratur bekannt. ${ }^{37)}$ Insofern könnte hier Einfluss genommen werden. Ob sich die Inhaber dieser Geschäfte freilich dazu bereitfinden, ist fraglich: Sie dürften die Waren so anzuordnen versuchen, dass ihr Umsatz bzw. Verdienst möglich gross ist. ${ }^{38)}$ Sollte der Staat andererseits eine solche Anordnung vorschreiben, kann man kaum mehr von sanftem Paternalismus sprechen, ganz abgesehen davon, dass hier ein erhebliches Kontrollproblem auftreten würde. Durch eine gezielte Anordnung der Speisen in öffentlich betriebenen Mensen und Cafeterien könnte man freilich ähnliche Effekte erzielen, ohne dass dagegen Einwände erhoben werden könnten. ${ }^{39)}$ Das in den Vereinigten Staaten sehr dringende Problem der übergewichtigen Kinder würde dadurch freilich nicht angegangen. Insgesamt aber scheinen die bis jetzt durchgeführten Expe-

34. Zu optimalen Sündensteuern die sich jedoch auf den Konsum von Kartoffelchips beziehen, siehe T. O'DonOGHUE und M. RABIN (2006).

35. Zu den Möglichkeiten des sanften Paternalismus, das Rauchen einzuschränken, siehe auch G. LUCAS (2012).

36. Siehe hierzu D. Cutler, A. Deaton und J.M. Shapiro (2003) für die Vereinigten Staaten und G. Brunello, P.-C. Michaud und A. SANZ-DE-GALdEANo (2009) für Europa.

37. Siehe z.B. X. DrÈze, St.J. Hoch und M.E. Purk (1994) oder P. CHANDON et al. (2009).

38. So finden z.B. H. DiXON, M. Scully und K. PARKINSON (2006) bei einer Untersuchung von Supermärkten in Melbourne, dass in der Nähe der Kassen besonders energiehaltige Lebensmittel platziert werden, die oft Kinder ansprechen sollen, damit die Eltern für sie diese kaufen. D.R. JUST und C.R. PAYNE (2009) sind dagegen der Überzeugung, dass es Möglichkeiten gibt, in diesem Sinn auf die Konsumenten Einfluss zu nehmen, ohne die Gewinnchancen der Unternehmen zu beeinträchtigen.

39. Siehe hierzu z.B. J.S. Downs, G. LoEwEnSTEIN und J. WISDOM (2009) sowie J. WISDOM, J.S. Downs und G. LOEWENSTEIN (2010). 
rimente keine Hoffnung auf grosse Erfolge in diesem Bereich zu rechtfertigen, wobei freilich auch streng paternalistische Massnahmen bisher kaum besser abgeschnitten haben. ${ }^{40)}$

[37] Ein Bereich, im welchem der sanfte Paternalismus ausgesprochen wirksam ist, der aber aus ethischer Perspektive sehr viel problematischer ist als die bisher angesprochenen Beispiele, ist jener der Organspende. ${ }^{41)}$ Auch hier dreht es sich wieder um die Vorgabe des Status Quo. Im (bisherigen) deutschen Modell gilt die (erweiterte) Zustimmungslösung: Ein Organ kann einem Verstorbenen nur dann entnommen werden, wenn dieser zu Lebzeiten (z.B. mittels eines Organspenderausweises) zugestimmt hat oder wenn die Angehörigen diese Zustimmung geben. Da diese Zustimmung innerhalb sehr kurzer Frist erfolgen muss, sehen sich die Angehörigen einer höchst problematischen Entscheidungssituation ausgesetzt. Das Ergebnis ist, dass nur vergleichsweise wenige Organe zur Verfügung stehen und in Deutschland jedes Jahr ca. 1000 Personen sterben, die auf ein Organ warten. ${ }^{42)}$

[38] Im Gegensatz dazu hat Österreich die Widerspruchslösung: Wer nicht ausdrücklich widersprochen hat, kommt als Organspender in Frage. Auch dies ist keine unproblematische Lösung, da es einen Eingriff in die Integrität des Menschen bedeutet, auch wenn dieser bereits tot ist. Ein derartiger Eingriff sollte - nach den bei uns vorherrschenden moralischen Vorstellungen - nur unter sehr restriktiven Bedingungen ohne Zustimmung der betroffenen Person erlaubt sein. Ob die Tatsache, dass damit ein Menschenleben verlängert werden kann, dazu hinreichend ist, ist umstritten. Tatsache aber ist, dass dadurch in Österreich etwa 50 Prozent mehr Transplantationen pro Kopf der Bevölkerung durchgeführt werden können. ${ }^{43)}$ Wie auch verschiedene wissenschaftliche Untersuchungen zeigen, hat die Frage, welche Regelung in einem Land gilt, signifikante Auswirkungen auf die Zahl der möglichen Transplantationen. Freilich zeigen diese Untersuchungen auch, dass dies nicht der einzige Einflussfaktor ist. ${ }^{44)}$

[39] Die neue deutsche Regelung, die im Juni 2012 im Bundesrat die letzte Hürde nahm, sieht vor, dass in Zukunft alle deutschen Bürgerinnen und Bürger regelmässig per Post von den Krankenkassen gefragt werden, ob sie sich zur Organspende bereitfinden würden. Dazu erhalten sie einen Organspenderausweis zugeschickt. Ähnlich wie bei der Zustimmungslösung handelt es sich um eine freiwillige Zustimmung, wobei jetzt jedoch ein starker Anreiz für die Betroffenen geschaffen wird, sich mit diesem Problem auseinanderzusetzen. Der ,Schubser` ist hier sehr viel schwächer als bei der Widerspruchslösung. Er entspricht freilich eher als diese der Philosophie des sanften Paternalismus, da die Betroffenen hier mit der relevanten Information direkt konfrontiert werden und es nicht geschehen kann, dass jemand, der sich nie damit befasst hat, gegen seinen Willen zum Organspender wird. Auch wenn das An-

40. Siehe hierzu z.B. G. Loewenstein, T. Brennan und K.G. Volpp (2007), D.R. Just und C.R. Payne (2009) sowie M.M. GALIZZI (2012). Zu den Erfahrungen in Grossbritannien siehe A. BuRGESS (2012).

41. Siehe hierzu z.B. R.H. ThAleR und C.R. Sunstein (2008, S. 240ff.). - Zur ethischen Problematik des ,Anstossens‘ siehe auch L. BOVENS (2009).

42. Siehe: Bundestag beschiesst Organspendereform, ZEIT-ONLINE vom 25. Mai 2012, http://www.zeit.de/ politik/deutschland/2012-05/organspende-bundestag (27/06/12).

43. Siehe hierzu Eurotransplant (2011, S. 36). - Dies bezieht sich freilich nur auf die über Eurotransplant abgewickelten Transplantationen, diejenigen, die z.B. im gleichen Spital durchgeführt werden, in dem auch die Entnahme stattfand, werden von dieser Statistik nicht erfasst.

44. Siehe hierzu z.B. A. AbAdiE und S. GAy (2006) oder A. Rhitalia et al. (2009). 
schieben hier vergleichsweise sanft geschieht, erhofft man sich davon eine deutliche Steigerung der Spendebereitschaft. Ob dies so eintreten wird, bleibt abzuwarten.

[40] Es wurden somit in den vergangenen Jahren eine ganze Reihe von Ansatzmöglichkeiten für Massnahmen des sanften Paternalismus aufgezeigt, von denen etliche auch realisierbar sind, und es gibt sicher noch eine ganze Reihe weiterer Möglichkeiten. Dennoch sollte man keine übersteigerten Erwartungen damit verbinden. Insbesondere legt das, was wir bisher über Massnahmen im Gesundheitsbereich wissen, Zurückhaltung nahe. Aber auch bei den 401(k)Plänen der Altersvorsorge sind die Unterschiede nicht so drastisch, wie es zunächst erscheinen mag: Schliesslich hat sich nach etwa 3 Jahren die Lücke in der Teilnahme zwischen denen die ablehnen konnten, und jenen die zustimmen mussten, geschlossen, wobei letztere freilich insgesamt mehr für ihre Altersvorsore gespart haben. Und das Beispiel der Organspende zeigt, dass mit solchen Massnahmen auch erhebliche ethisch-moralische Probleme verbunden sein können.

\section{$5 \quad$ Zur Kritik am sanften Paternalismus}

[41] Am Konzept des sanften Paternalismus wurde selbstverständlich auch Kritik geübt. Auch wenn einige der Einwände sicherlich berechtigt erscheinen, so erstaunt doch die Schärfe, mit der diese Kritik teilweise vorgebracht wird. Dies mag mit dem vom sanften Paternalismus erhobenen Anspruch zusammenhängen, eben nicht - wie der traditionelle Paternalismus - in die Präferenzen der Individuen eingreifen zu wollen, sondern diese zu respektieren und ausschliesslich Hilfen zu bieten, damit es den Betroffenen leichter fällt, sich gemäss ihrer ,wahren` Präferenzen zu entscheiden.

[42] Zunächst wird z.B. von J. SchnellenBACH (2012) die (wohlfahrtsökonomische) Fundierung des sanften Paternalismus infrage gestellt. Er argumentiert, dass es nicht sicher sei, dass die vorgeschlagenen Massnahmen effizienzsteigernd wirken, da das umfassende Wissen nicht zentral vorhanden wäre, welches erforderlich wäre, um eine Pareto-Verbesserung zu garantieren. (S. 268ff.) Sie könnten mit nicht-kalkulierbaren, nicht intendierten Nebenfolgen verbunden sein. Auch werde die Autonomie der Individuen eingeschränkt, die für ihre subjektive Zufriedenheit wesentlich sei. Da die Massnahmen notwendigerweise mit Umverteilung verbunden seien, könnten Minderheiten durch eine von ihnen nicht erwünschte Verteilungspolitik negativ betroffen werden. Eine negative Betroffenheit kann sich nach H. BECK (2010, S. 53) auch dadurch ergeben, dass Menschen mit anderen Präferenzen, wie z.B. Raucher, durch staatliche Massnahmen wie z.B. Aufklärungskampagnen mit einer ,psychologischen Steuer` belegt werden.

[43] G. Whitman (2006) wirft den Vertretern des libertären Paternalismus vor, dass sie mit ihrem Konzept dort stünden, wo die Theorie der Externalitäten vor 1960 stand, d.h. bevor Ronald Coase seine berühmte Arbeit über die Möglichkeit der Internalisierung externer Effekte durch Verhandlungen veröffentlicht hatte. Sie würden bestimmte private Interessen über die anderer stellen, sie würden Verhandlungen innerhalb der Person, d.h. zwischen dem Selbst mit der kurz- und jenem mit der langfristigen Präferenzfunktion genauso wenig beachten wie die Möglichkeiten der Individuen, eigene Verfahren zur Überwindung der Zeitinkonsistenz zu 
entwickeln, und sie würden auch die Möglichkeit des Politikversagens nicht in Betracht ziehen.

[44] Sanfter Paternalismus soll zudem das Lernen der Individuen beeinträchtigen. So kritisiert z.B. J. SCHNELLENBACH (2012), dass durch sanften Paternalismus die Anreize der Individuen, durch Versuch und Irrtum zu lernen, abgeschwächt würden. ${ }^{45)}$ E.L. GLAESER (2006) argumentiert, dass durch den libertären Paternalismus für die Individuen die Anreize, selbst etwas gegen die Willensschwäche zu tun, abgeschwächt würden, weshalb er in der Existenz der Entscheidungsanomalien eher Argumente für eine stärkere Begrenzung als für eine Ausdehnung der Staatsaktivität sieht. J. SchNELLENBACH (2011) bemerkt in diesem Zusammenhang, dass es einen Markt für Selbstbindungen gebe, in welchem man dann, wenn man wolle, Selbstbindungen eingehen könne, die im Allgemeinen treffsicherer und eher wohlfahrtssteigernd wären. Er sieht kollektive Selbstbindungen allenfalls für echtes Suchtverhalten als sinnvoll an.

[45] Dabei wird von J. SchnelLENBACH (2012) auch in Zweifel gezogen, dass die übergeordneten, langfristigen Präferenzen tatsächlich die ,wahren' Präferenzen sind, an denen sich die Individuen ausrichten sollten, da sie dann, wenn sie ihren aktuellen Präferenzen folgen, sich der Kosten ihrer Handlungen eher bewusst sind, und die langfristigen eher ,expressiv sind. ${ }^{46)}$ Ähnlich argumentiert wiederum E.L. GLAESER (2006), wenn er darauf verweist, dass die Individuen als Wähler geringere Anreize haben, sich ,richtig‘ zu entscheiden, als bei wirtschaftlichen Entscheidungen. ${ }^{47)}$

[46] R. SugDEN (2008) kritisiert, dass bei den Vorschlägen zum sanften Paternalismus, nicht klar sei, wie bzw. durch wen sie durchgesetzt werden sollen. Mehrere Kritiker unterstellen, dass diese Vorschläge sich an einen allwissenden Planer richten bzw. einen wohlwollenden Diktator voraussetzen. Dies bedeute nicht nur eine Anmassung an Wissen, wie sie bereits von F.A. V. HAYEK (1945) kritisiert wurde. ${ }^{48)}$ Es setze auch voraus, dass diese Planer nicht den gleichen Verhaltensanomalien unterliegen wie die ,normalen“ Individuen, die durch die Anstösse, die von den Planer ausgehen, ,geschubst` ${ }^{`}$ werden sollen. ${ }^{49)}$ Ausserdem biete der sanfte Paternalismus, worauf H. BECK (2010) hinweist, Einfallstore für Missbrauch und Manipulation. Dabei seien diese sanften Anstösse, wie E. Seliger und K.P. WhYTe (2012) bemerken, kaum in der Lage, komplexe Politikprobleme zu lösen. Dann aber müsse auch der libertäre Paternalismus zu ,härteren‘ Massnahmen greifen, d.h. er sei nicht mehr sanft. Ganz allgemein bestehe die Gefahr, dass man - beginnend mit ,sanften` Massnahmen - sich auf eine schiefe Ebene begebe und schliesslich beim ,harten` Paternalismus lande. ${ }^{50)}$

45. Ähnlich argumentieren auch B.I. CARLin, S. Gervais und G. MANSO (2011), die freilich - im Gegensatz zu J. SCHNELLENBACH $(2011,2012)$ - nicht grundsätzlich gegen den liberalen Paternalismus argumentieren.

46. Siehe hierzu auch G. BRENNAN und L. LOMASKY (1985). J. SCHNELLENBACH (2012) bestreitet den Vorzug der übergeordneten Präferenzen auch deshalb, weil man nicht wisse, wie lang man lebe, weshalb es möglicherweise sinnvoll sei, den kurzfristigen Präferenzen den Vorzug zu geben.

47. Dieses Argument geht auf J.M. BuCHANAN (1954) zurück.

48. Siehe z.B. M.J. Rizzo und G. Whiteman (2009, S. 910).

49. Siehe z.B. G. Whiteman und M.J. Rizzo (2007, S. 442f.) sowie M.J. Rizzo und G. Whiteman (2009a, S. 723ff.).

50. Siehe hierzu z.B. M.J. Rizzo und G. Whiteman (2009a) sowie G. Whiteman und M.J. RizzO (2007.) 
[47] Ein Teil dieser Einwände ist berechtigt. So sind manche Erwartungen, die in die Massnahmen des sanften Paternalismus gesetzt werden, überzogen. Dies zeigen nicht zuletzt die oben berichteten Ergebnisse des Einsatzes solcher Massnahmen im Gesundheitsbereich. Die Tatsache, dass Entscheidungsanomalien auftreten, bedeutet auch nicht, dass der Staat eingreifen muss. Dies würde auch kein Vertreter des sanften bzw. libertären Paternalismus fordern. Mögliches Politikversagen wird sehr wohl in Rechnung gestellt. Andererseits existiert heute nicht nur ein sanfter, sondern sogar ein recht harter Paternalismus, der mit Anreizen, d.h. Steuern und Subventionen, aber auch Ge- und Verboten arbeitet. Man sollte das Konzept des sanften Paternalismus daher nicht mit einer (vielleicht) idealen Situation ohne jeglichen Paternalismus vergleichen, da man sonst wieder in die - von der institutionellen Ökonomik zu Recht verabschiedete - Diskussion im Nirwana gerät. Die entscheidende Frage (bzw. das Anliegen der Vertreter des libertären Paternalismus) ist vielmehr, wie man paternalistische Regelungen, die so oder so existieren, so ausgestalten kann, dass sie ,sanfter` als die heutigen Regelungen werden, d.h. dass sie weniger in die freien Entscheidungen der Individuen eingreifen. Hoffnungen, durch sanfte Massnahmen alle härteren paternalistischen Massnahmen ablösen zu können, dürften freilich illusorisch sein. Man kann bestenfalls hoffen, einige der härteren durch sanftere Massnahmen zu ersetzen.

[48] Andere Kritikpunkte dürften weit überzogen sein. Dies gilt für weite Teile der Kritik an der (fehlenden) wohlfahrtsökonomischen Fundierung. Wie oben ausgeführt wurde, bietet das Modell hierarchisch geordneter Präferenzen sehr wohl eine solche Fundierung. Auch wenn es in einigen Bereichen Möglichkeiten individueller Selbstbindungen gibt: Dies impliziert nicht, dass deshalb kollektive Selbstbindungen über den politischen Prozess nicht zulässig wären. Man kann dies den Bürgerinnen und Bürgern im demokratischen System ja nicht verbieten. Schliesslich ist es dort nicht eine abgehobene Obrigkeit, sondern es sind die Bürgerinnen und Bürger, die entweder selbst (in der direkten Demokratie) oder durch ihre Repräsentanten (im indirekten System) über solche Massnahmen entscheiden. Das Problem ist dabei kaum, wie E.L. GLAESER (2006) glaubt, dass die Individuen in ihrer Rolle als Stimmbürger weniger verantwortungsbewusst handeln als in ihrer Rolle als Wirtschaftsbürger. Schliesslich gibt es Evidenz dafür, dass sie bei Abstimmungen die Interessen anderer stärker berücksichtigen als auf dem Markt. ${ }^{51)}$

[49] Interessant sind in diesem Zusammenhang auch die Bemerkungen von J. SchNELLENBACH (2012, S. 274), wo er den Wählerinnen und Wähler vorwirft, dass ihre Entscheidung nicht „ein wahres materielles Eigeninteresse der Wähler“ abbildet, und er wirft dem demokratischen Prozess vor, dass eine Mehrheit Regelungen befürworten kann, „die nicht im materiellen Eigeninteresse einer Mehrheit der Wähler liegen.“ Demokratische Politik tendiere dazu, „das Ausmass der bereits existierenden kognitiven Verzerrungen zu vergrössern.“ Hier bestätigt er nicht nur das - für die moderne Wirtschaftswissenschaft in aller Regel nicht mehr angebrachte, aber immer noch weit verbreitete - Vorurteil, dass im Rahmen des ökonomischen Ansatzes nur materielle Werte berücksichtigt werden und alles andere wie z.B. moralische

51. Siehe hierzu G. KIRChGÄSSner (2010) sowie W.W. POMMEREHNE und F. SCHNEIDER (1985), die für den Kanton Basel-Landschaft gezeigt haben, dass bei Abstimmungen über die erste Säule der Schweizerischen Alterssicherung, der Alters- und Hinterlassenenversicherung (AHV), Mehrheiten dadurch zustande kamen, das Stimmberechtigte gegen ihre eigenen materiellen Interessen gestimmt haben. 
oder ethische Werte nicht zählt, und dass überdies Eigeninteresse das ausschliessliche Kriterium ist, an welchem sich rationale Individuen orientieren sollten. Dass das Funktionieren einer modernen demokratischen Gesellschaft u.a. davon abhängt, dass eine Mehrheit der Bürgerinnen und Bürger in bestimmten Situationen ihre eigenen Interessen zugunsten von Regelungen zurückstellt, welche die Stabilität der Gesellschaft sichern, wird völlig ausgeblendet. ${ }^{52}$ )

[50] Auch die Behauptung, dass die kurzfristigen eher als die langfristigen den wahren Präferenzen entsprechen, ist wenig überzeugend. Angesichts der Ergebnisse im Labor wie im Feld impliziert dieses Argument eine extrem hohe ,richtige‘ Diskontrate. Damit kann man freilich, wie G.S. BECKER und K.M. MURPHY (1988) gezeigt haben, selbst extremes Suchtverhalten als rationale Entscheidung mündiger Individuen darstellen. Da man die ,wahren‘ Präferenzen nicht objektiv feststellen kann, kann man freilich weder beweisen, dass die kurzfristigen, noch dass die langfristigen eher den wahren Präferenzen entsprechen. Die Tatsache, dass die Menschen dann, wenn sie über sich selbst reflektieren, in aller Regel eine langfristige Perspektive einnehmen, spricht eher für die langfristigen Präferenzen. Das gleiche gilt für die Beobachtung, dass sie bei ihren durch die kurzfristigen Präferenzen gesteuerten Handlungen die längerfristigen Kosten (und Nutzen) häufig massiv unterschätzen, wie dies auch die oben referierten Ergebnisse der Verhaltensökonomik zeigen. Das Problem der Willensschwäche besteht ja nicht in einer Unterschätzung der kurzfristigen Kosten einer Handlung, sondern als Folge einer sehr hohen kurzfristigen Diskontrate - in der massiven Unterschätzung ihrer langfristigen Konsequenzen zum Zeitpunkt einer Handlung. Andernfalls könnte man z.B. auch das Bedauern über eigene frühere Handlungen, welches dann auftritt, wenn vorhersehbare, aber nicht beachtete negative Konsequenzen deutlich geworden sind, nicht verstehen.

[51] Die von J. Schnellenbach (2012) geäusserte Kritik, dass man bei Massnahmen des libertären Paternalismus nie sicher sein könne, näher an den Nutzenrand einer Gesellschaft zu kommen, ist ebenfalls wenig überzeugend. Sie ist trivialerweise insofern korrekt, als es kaum eine wirtschaftspolitische Massnahme gibt, die nur Gewinner kennt und damit eine ParetoVerbesserung garantiert. Aus dieser Perspektive lässt sich allenfalls ein Minimalstaat im Sinne von F.A. V. HAYEK (1960) oder R. NOZICK (1974) rechtfertigen, auch wenn die dort versuchten Begründungen für ein solches Konzept sehr umstritten sind. Tatsächlich aber kommt es nicht darauf an, dass eine Pareto-Verbesserung gewährleistet werden kann: Dies wäre nur in einer - nicht existierenden - Welt vollständiger Voraussicht möglich. Wenn man überhaupt mit dem Pareto-Kriterium arbeiten will, geht es darum, ob eine Pareto-Verbesserung erwartet werden kann. Hierzu ist nicht vollständige Voraussicht erforderlich, sondern ,nur', dass die Individuen Erwartungen bilden können und diese aufgrund ihrer Erfahrungen anpassen, d.h. dass sie lernen. ${ }^{53)}$

52. Siehe hierzu G. KIRCHGÄSSNER (2008, S. 154ff.).

53. Folgt man der Argumentation, dass der Staat nur solche Handlungen unternehmen sollte, die zu einer Pareto-Verbesserung führen, dürfte er auch keine Information z.B. zur Behebung von Informationsasymmetrien bereitstellen bzw. Institutionen finanziell unterstützen, die solche Informationen bereitstellen, wie in Deutschland z.B. die Stiftung Warentest. Deren Kosten müssen vom Steuerzahler aufgebracht werden, und damit müssen sich auch Bürgerinnen und Bürger an der Finanzierung beteiligen, denen aus diesen Aktivitäten kein Nutzen erwähnt. Sie werden somit durch solche staatlichen Aktivitäten schlechter gestellt, womit das Pareto-Kriterium verletzt ist. - Siehe hierzu auch P. D. MARNEFFE (2006), der keinen Sinn darin sieht, bei staatlichen Massnahmen grundsätzlich nach Erklärungen zu suchen, die nicht auf Paternalismus rekurrieren, da dadurch gelegentlich die besten Argumente für diese Massnahmen ausgeschlossen würden. 
[52] Entscheidungen über paternalistische Massnahmen werden im demokratischen Staat freilich mit Mehrheit getroffen, weshalb sie in aller Regel zumindest einen Teil der Bevölkerung mit Kosten belasten. Daher sollte idealerweise auf der Verfassungsebene, d.h. hinter dem Schleier der Ungewissheit festgelegt werden, in welchen Bereichen solche Regelungen getroffen werden dürfen und wie weit sie gehen sollen. Natürlich kann man darüber spekulieren, welche Regeln in einer solchen Situation festgelegt würden, wie dies z.B. J.M. BUCHANAN und G. TUllOCK (1962), J. RAWLS (1971) oder J.M. BuCHANAN (1975) in ihren Verfassungstheorien getan haben. Das Problem ist freilich, dass wir eine solche Situation im Allgemeinen nur sehr bedingt und unvollständig herstellen können ${ }^{54)}$ und dass die Meinungen darüber, welche Regeln in einer solchen Situation beschlossen würden, weit auseinander gehen. ${ }^{55}$ Deshalb für sich aber das Recht zu reklamieren, autoritativ darüber bestimmen zu dürfen, welche Entscheidungen die Bürgerinnen und Bürger kollektiv über den politischen Prozess treffen dürfen und welche sie dem Markt überlassen sollten, steht dem Wissenschaftler nicht zu, auch wenn er sich zu einer liberalen Grundhaltung bekennt. Sonst setzt er sich dem gleichen Vorwurf aus, anderen seine eigene Wertordnung aufoktroyieren zu wollen, mit dem er anderen, z.B. den Vertretern des libertären Paternalismus, begegnet. ${ }^{56)}$

[53] Interessant ist auch die Behauptung von J. ScHnELlEnBACH (2012), dass libertärer Paternalismus (zumindest in der Tendenz) den Status Quo stabilisiere. Die Argumentation läuft darüber, dass die entsprechenden Massnahmen sich an den herrschenden gesellschaftlichen Normen orientieren und deren Gültigkeit damit gestärkt werde. Nun wollen die libertären Paternalisten den Status Quo in wichtigen gesellschaftlichen Bereichen, wie z.B. dem Gesundheitsbereich oder der Altersvorsorge, gerade abändern. Insofern klingt dieser Vorwurf etwas merkwürdig, auch wenn nicht zu bestreiten ist, dass sie damit gewissen normativen Vorstellungen darüber, wie ein gesundes Essverhalten aussieht bzw. wie wir sinnvollerweise für unser Alter vorsorgen sollten, stärker zum Durchbruch verhelfen wollen.

[54] Andererseits ist eine Stabilisierung des Status Quo nicht grundsätzlich schlecht. Im politischen Raum erscheint er uns ihn in bestimmten Bereichen so wichtig zu sein, dass wir ihn in der Verfassung dadurch absichern, dass wir für Änderungen qualifizierte Mehrheiten verlangen. Aber auch in der normativen ökonomischen Theorie spielt der Status Quo eine herausragende Rolle. ${ }^{57)}$ Dies gilt insbesondere deswegen, weil das konservativste aller Wohlfahrtskriterien, das Pareto-Kriterium, ihr in aller Regel zugrunde gelegt wird. Auch J. ScHNELLENBACH (2012) beruft sich in seiner Kritik am sanften Paternalismus auf dieses Kriterium und verteidigt damit zumindest implizit den Status Quo. Insofern ist seine diesbezügliche Kritik am sanften Paternalismus bemerkenswert.

[55] Ambivalent ist auch das Argument von E.L. GLAESER (2006), dass durch die sanften Anstösse bei den betroffenen Individuen die Anreize zum Lernen abgeschwächt werden. Dies

\footnotetext{
54. Siehe hierzu G. KIRCHGÄSSNER (1994).

55. Siehe hierzu z.B. die Diskussion der von J. RAwLS (1971) vorgeschlagenen Gerechtigkeitsprinzipien in N. DANIELS (1975) sowie in American Political Science Review 69 (1975), Heft 5, S. 585ff.
}

56. Siehe z.B. J. SCHNELLENBACH (2011, S. 452).

57. Siehe hierzu die Beiträge in Constitutional Political Economy 15 (2004), Heft 2, insbesondere die Einleitung von G. BRENNAN (2004). 
mag im Einzelfall richtig sein, besagt aber nicht wirklich etwas. Die Existenz der Entscheidungsanomalien zeigt ja gerade, dass das Lernen der Individuen nicht hinreichend ist, sie zu vermeiden. Wenn sie dies selbst und die sich daraus für sie selbst ergebenden negativen Konsequenzen erkennen, bedeutet es nicht, dass sie ihnen beim nächsten Mal nicht mehr zum Opfer fallen. Insofern können sie gerade dann, wenn sie erkannt haben, dass es sich hier um eine Anomalie handelt, befürworten, dass solche Anstösse erfolgen, welche diese negativen Konsequenzen zu vermeiden helfen. Und ganz allgemein gilt, dass die Frage, inwieweit es sinnvoll ist, selbst zu lernen, und inwieweit wir sicherstellen sollten, dass wir aus der Erfahrung anderer lernen, situationsabhängig ist. Es ist nicht grundsätzlich besser, wie J. SCHNELLENBACH (2012) suggeriert, selber durch Versuch und Irrtum zu lernen; man kann sich auch die Erfahrungen anderer zunutze machen.

\section{Zusammenfassung und Abschliessende Bemerkungen}

[56] Politische Eingriffe in Marktprozesse werden in aller Regel durch die Existenz externer Effekte und/oder öffentlicher Güter gerechtfertigt. Dabei spielen die Rechte und Interessen Dritter eine zentrale Rolle: Es geht darum, negative Auswirkungen der Aktivitäten Dritter auf die Individuen auszugleichen (negative externe Effekte) bzw. positive Auswirkungen auf Dritte zu kompensieren. Im Gegensatz dazu spielen die Rechte und Interessen Dritter beim Paternalismus keine Rolle: Sie werden gar nicht tangiert. Es geht vielmehr darum, in die Entscheidungen der betroffenen Bürgerinnen und Bürger zu deren eigenem Wohl einzugreifen. In den Wirtschaftswissenschaften werden solche Bemühungen seit R.A. MuSGRAVE (1957) unter dem Begriff der ,Meritorik‘ diskutiert.

[57] Individuen in Widerspruch zu ihren geäusserten Präferenzen zu ihrem eigenen Wohl beeinflussen zu wollen, steht zumindest dem ersten Anschein nach im Widerspruch zur traditionellen Auffassung des normativen Individualismus, wonach die (autonomen) Individuen ausschliesslich Träger der Werte sind und niemandem das Recht zusteht, ihnen die Werte, nach denen sie handeln wollen, vorzuschreiben. Diese Auffassung liegt auch der (traditionellen) normativen Ökonomik, der Wohlfahrtstheorie, zugrunde; sie konkretisiert sich dort im Konzept der ,Konsumentensouveränität‘. Paternalismus muss freilich dann nicht im Widerspruch zum normativen Individualismus stehen, wenn wir - der philosophischen Tradition folgend - davon ausgehen, dass die Individuen in der Lage sind, über ihr eigenes Tun zu reflektieren, d.h. dass sie über hierarchisch geordnete Präferenzordnungen verfügen, bei denen sie mit Hilfe der übergeordneten, reflexiven Präferenzen ihre jeweils aktuellen Präferenzen, gemäss deren sie in konkreten Situationen handeln, einschätzen können. Paternalistische staatliche Massnahmen können dann als über den politischen Prozess erreichte kollektive Selbstbindungen begriffen werden. Auch wenn sich damit vermutlich nicht alle heute ergriffenen paternalistischen Massnahmen rechtfertigen lassen, ist damit eine generelle Ablehnung jeden Paternalismus aufgrund dessen angeblicher Unvereinbarkeit mit dem normativen Individualismus nicht mehr haltbar.

[58] Während der traditionelle, ,harte‘ Paternalismus mit Ge- und Verboten sowie mit Anreizen, insbesondere Steuern und Subventionen arbeitet, will der sanfte bzw. libertäre Paternalismus solche direkten Eingriffe vermeiden und nur mit sanften Anschüben, ,Nudges‘, arbeiten. Grundlage für solche Massnahmen sind Erkenntnisse der Verhaltensökonomik, die zei- 
gen, dass Menschen trotz objektiv gleicher Information ganz unterschiedlich auf eine Herausforderung reagieren, je nachdem wie die Information aufbereitet wird (,Framing`) bzw. was als Status Quo vorgegeben wird. Der sanfte Paternalismus arbeitet damit im Wesentlichen mit einer gezielten Aufbereitung der verfügbaren Information. Das ,libertäre‘ an diesem Konzept besteht darin, dass die Menschen in eine bestimmte Richtung gelenkt werden sollen, ohne dass ihre Entscheidungsfreiheit dadurch beeinträchtigt wird.

[59] Es gibt eine ganze Reihe politischer Massnahmen, die im Rahmen dieses Konzepts vorgeschlagen wurden. Sie beziehen sich insbesondere auf die Altersvorsorge sowie auf den Gesundheitsbereich; hier werden insbesondere die Probleme des Übergewichts, des Rauchens sowie der Organspende thematisiert. Die vorgeschlagenen Massnahmen arbeiten u.a. damit, dass in einer bestimmten Situation eine bestimmte Handlungsalternative, die aus einer bestimmten Perspektive erwünscht erscheint, als Regel vorgegeben wird. Man kann von ihr ohne Probleme abweichen. Dies erfordert freilich eine aktive Entscheidung.

[60] Betrachtet man die vorgeschlagenen Massnahmen genauer, wird zweierlei deutlich. Soweit sie überhaupt praktikabel sind, sind die Vorschläge zum einen zum Teil wenig wirksam. Dies gilt insbesondere für viele der für den Gesundheitsbereich vorgeschlagenen Massnahmen. Zweitens aber kann mit ihnen bestenfalls ein geringer Teil der heute in Kraft befindlichen harten paternalistischen Massnahmen ersetzt werden. Dazu kommt, dass der Übergang zwischen traditionellem und sanftem Paternalismus fliessend ist: Zumindest einige der vorgeschlagenen Massnahmen laufen auch auf eine Veränderung der relativen Preise hinaus. Dies wird z.B. deutlich, wenn für den Gesundheitsbereich ,Sündensteuern' diskutiert werden. Insofern unterscheidet sich der sanfte nur graduell vom traditionellen Paternalismus, weshalb jene Argumente, die gegen den traditionellen Paternalismus vorgebracht werden, in gleicher Weise auch gegen den sanften Paternalismus vorgebracht werden können.

[61] Die (ökonomischen) Kritiker des sanften Paternalismus gehen von einer (in ihrer Vorstellung) idealen Welt ohne Paternalismus aus. Dies gilt insbesondere dort, wo argumentiert wird, dass die Vorschläge des sanften Paternalismus die Situation verschlimmern würden. Tatsächlich aber leben wir heute in einer Welt, in der Paternalismus allgegenwärtig ist, egal ob es sich um obligatorische Sozialversicherungen, Subventionen für Theater und Oper, kostenlose Vorsorgeuntersuchungen oder die Anschnallpflicht im Kraftfahrzeug handelt. Welche Wirkung Massnahmen des sanften Paternalismus tatsächlich haben würden, kann aber nur durch einen Vergleich mit der heutigen, tatsächlichen Situation herausgefunden werden. Dabei ist insbesondere danach zu fragen, welche bisher existierenden Massnahmen des harten Paternalismus dadurch möglicherweise abgelöst werden könnten.

[62] Ein radikaler Anti-Paternalismus, wie er von einigen Ökonomen vertreten wird und wie er sich auf JoHn StUART MiLl berufen kann, der z.B. die Ablehnung (fast) aller Arbeitsschutzgesetze implizieren würde, ist vermutlich wenig sinnvoll und kaum durchhaltbar. Vor allem aber dürfte er im (demokratischen) politischen Prozess kaum durchsetzbar sein. Dies wird vor allem dort deutlich, wo meritorische Politik durch Volksabstimmungen legitimiert wird.

[63] Ein radikaler Anti-Paternalismus ist auch deshalb nicht möglich, weil man dann, wenn man erkannt hat, dass die Aufbereitung der Information Auswirkungen auf die Entscheidun- 
gen der Individuen hat, sich der Frage stellen muss, wie die Information aufbereitet werden soll. Wann immer die Rückfalloption eine Rolle spielt, beeinflussen der Staat oder das entsprechende Unternehmen durch die Vorgabe der ,normalen“ Regelung das Verhalten der Betroffenen. Das Gleiche gilt, wenn das Verhalten vom gewählten Rahmen abhängig ist. In diesen Situationen dürfte es Sinn machen, sich zu überlegen, welcher Rahmen gewählt werden soll bzw. was die Rückfalloption sein soll. Die Augen davor zu verschliessen, dass hier in jedem Fall eine Entscheidung getroffen wird oder einfach das weiterzuführen, was man bisher getan hat, dürfte keine rationale Strategie sein. Zudem gilt hier auch nicht das schon ansonsten fragwürdige Argument, dass die kurzfristige mehr Gewicht haben sollte als die langfristige Präferenzfunktion. Schliesslich scheinen hier in Abhängigkeit von der Rückfalloption unterschiedliche kurzfristige Präferenzfunktionen zum Einsatz zu kommen (bzw. die gleiche kurzfristige Präferenzfunktion führt in Abhängigkeit von der Rückfalloption zu völlig unterschiedlichen Ergebnissen), weshalb man dann, wenn man nur die kurzfristigen Entscheidungen betrachtet, nicht sagen kann, was ,besser` und was ,schlechter` ist. Die kurzfristige Präferenzfunktion hält hierfür keine Kriterien bereit. Andererseits erscheint es gerade dann, wenn ein solcher ,Konflikt‘ zwischen kurzfristigen Präferenzfunktionen bzw. zwischen Handlungsweisen, die sich, gegeben die kurzfristige Präferenzfunktion, aus unterschiedlichen Frames ergeben, sinnvoll zu sein, auf die langfristigen Präferenzen zu rekurrieren.

[64] Dass sich die Kritiker des sanften Liberalismus häufig auf F.A. V. HAYEK berufen, dürfte kein Zufall sein; schliesslich ist er ein extremer Kritiker des staatlichen Interventionismus, wozu meritorische Politik in besonderer Weise zählt. Dabei wird in aller Regel gar nicht berücksichtigt, dass Entscheidungen über meritorische Politikmassnahmen in demokratischen Systemen nicht von einem zentralen Planer, sondern (in der direkten Demokratie) von den Bürgerinnen und Bürgern selbst bzw. (im repräsentativen System) von deren Repräsentanten getroffen werden, die sich aber zumindest begrenzt an den Präferenzen der Bürgerinnen und Bürger orientieren müssen. Bekennt man sich zum demokratischen Prinzip, geht es also letztlich darum, diejenigen politischen Massnahmen zu ergreifen, welche die Bürgerinnen und Bürger wünschen, und dies sollte unabhängig davon sein, inwieweit diese Massnahmen mit einer bestimmten Ideologie vereinbar sind. Man kann die Vereinbarkeit oder Nichtvereinbarkeit mit einer bestimmten politischen Doktrin zwar als Argument in die Diskussion politischer Massnahmen einbringen, aber wenn die Bürgerinnen und Bürger z.B. Mechanismen der kollektiven Selbstbindung wünschen, kann man ihnen in einer Demokratie nicht verbieten, solche kollektiven Lösungen anzustreben. Man kann, und das ist - im Gegensatz zur Vorgabe von Regeln - die Aufgabe der Verfassungsökonomie, aufzeigen, bei der Anwendung welcher Regeln welche Ergebnisse zu erwarten sind. Die Festlegung der Regeln muss man - im demokratischen Staat - schon den Bürgerinnen und Bürgern selbst überlassen. ${ }^{58)}$ Die oben bereits erwähnte Tatsache, dass die Entscheidungen über diese Regeln wenn überhaupt, dann nur unter einem sehr dünnen Schleier der Unwissenheit getroffen werden, ändert daran nichts. ${ }^{59)}$

58. Siehe hierzu auch K. SCHMIDT (1988).

59. Siehe hierzu auch CH. CORDES und CH. SCHUBERT (2011). 
[65] Im Gegensatz zu den anderen Kritikern sieht J. SCHNELlEnBaCH (2011, 2012) zwar, dass hier ein demokratischer politischer Prozess zugrunde liegt, aber wie oben ausgeführt wurde, kritisiert er daran, dass die Wähler bzw. Stimmbürger nicht ausschliesslich ihren „wahren materiellen Interessen“ (2012, S. 274) folgen. Diese Kritik an der Demokratie liegt in der Tradition von F.A. v. HAYEK, der dem demokratischen Prozess ausgesprochen skeptisch gegenüber stand: Er zog, wie er selbst schrieb, „eine beschränkte nicht-demokratische Regierung einer nicht-beschränkten demokratischen ... vor.“ (1977, S. 9). In dieser Tradition steht z.B. auch B. CAPLAN, der der Auffassung ist, „die Demokratie scheitert, weil sie das tut, was die Wählerinnen und Wähler wünschen“ (2007, S. 3), und dieser Auffassung scheint sich auch J. SCHNELLENBACH (2012) anzuschliessen. Ob wir uns freilich dann besser stellen würden, wenn wir über die Organisation unserer politischen Prozesse nicht mehr selbst entscheiden dürften, sondern dies den wohlwollenden Diktatoren der Verfassungsökonomik überlassen müssten, die zwar allen anderen unterstellen dass sie ihren eigenen Nutzen maximieren, aber für sich selbst als Ausnahme reklamieren, dass sie die allgemeine Wohlfahrt anstreben, sei dahingestellt. Verglichen damit ist der Anspruch des sanften Paternalismus, so sehr man ihn auch - und durchaus zu Recht - kritisieren kann, eher bescheiden.

\section{Zusammenfassung}

Paternalistische bzw. meritorische Politik versucht, auf die Entscheidungen der Individuen zu deren eigenem Wohl Einfluss zu nehmen, auch wenn keine Dritten davon betroffen sind. Dies scheint im Gegensatz zum normativen Individualismus zu stehen, der als Leitbild unserer modernen Demokratie zugrunde liegt. Der sanfte Paternalismus sieht durch die Existenz von Verhaltensanomalien die Notwendigkeit paternalistischer Politik begründet, möchte aber hier mit sehr niedrigschwelligen Massnahmen arbeiten, die insbesondere die Entscheidungsmöglichkeiten der Betroffenen offen lassen. Tatsächlich unterliegt er jedoch den gleichen möglichen Einwänden wie die ,harte' Variante. Sobald wir jedoch davon ausgehen, dass die Menschen über ihr Tun und auch über ihre jeweils handlungsleitenden Präferenzen reflektieren können, widerspricht Paternalismus nicht mehr notwendigerweise dem normativen Individualismus. Die Einsatzmöglichkeiten des sanften Paternalismus sind, soweit man das bisher beurteilen kann, jedoch eher begrenzt. Die vielfach geäusserte Kritik an diesem Konzept ist teilweise berechtigt, schiesst aber teilweise auch weit über das Ziel hinaus und scheint zum Teil auch eher ideologisch motiviert zu sein. 


\section{Literaturangaben}

A. VAN AAKEN, (2006), Begrenzte Rationalität und Paternalismusgefahr: Das Prinzip des schonendsten Paternalismus, in: K. Seelmann, A. Anderheiden, P. BÜRKLI, M. Heinig und S. KIRSTE (eds.), Paternalismus und Recht, Mohr Siebeck, Tübingen 2006, S. 109 - 144.

A. ABADIE und S. GAY (2006), The Impact of Presumed Consent Legislation on Cadaveric Donation: A Cross-Country Study, Journal of Health Economics 25 (2006), S. 599 - 620.

N. ANDEL (1984), Zum Konzept der meritorischen Güter, Finanzarchiv 42 (1984), S. 630 - 648.

ARISTOTELES, Nikomachische Ethik; deutsche Übersetzung: Akademie Verlag, 9. Auflage, Berlin 1991.

R.J. ARNeSON (2005), Joel Feinberg and the Justification of Hard Paternalism, Legal Theory 11 (2005), S. $259-284$.

H. BECK (2010), Ist paretianischer Liberalismus doch möglich? Verhaltenswissenschaftliche Ökonomik und Liberaler Paternalismus, List Forum für Wirtschafts- und Finanzpolitik 36 (2010), S. 43 58.

G.S. BeCKer und K.M. MurPhy (1988), A Theory of Rational Addiction, Journal of Political Economy 96 (1988), S. 675 - 700.

B.D. Bernheim, A. FradKin und I. Popov (2011), The Welfare Economics of Default Option: A Theoretical and Empirical Analysis of 401(k) Plans, NBER Working Paper Nr. 17587, November 2011.

L. Bovens (2009), The Ethics of Nudge, in: T. GRÜNE-YANOFF und S.O. HANSEN (eds.), Preference Change: Approaches from Philosophy, Economics and Psychology, Springer, Dordrecht et al. 2009, S. $207-219$.

G. Brennan und A. Hamlin (2004), An Introduction to the Status Quo, Constitutional Political Economy 15 (2004), S. 127 - 132.

G. BREnNAN und L. LOMASKY (1984), Institutional Aspects of Merit Goods Analysis, Finanzarchiv N.F. 41 (1984), S. $183-206$.

G. Brunello, P.-C. Michaud und A. SAnZ-DE-GaldeAno (2009), The Rise of Obesity, Economic Policy 24 (2009), S. 551 - 596.

J.M. BuChanAn (1975), The Limits of Liberty, University of Chicago Press, Chicago 1975; deutsche Übersetzung: Die Grenzen der Freiheit, Mohr (Siebeck), Tübingen 1984.

J.M. Buchanan (1982), Kommentar, in: P. KosLOWsKi, Ethik des Kapitalismus, Mohr (Siebeck), Tübingen, 6. Auflage 1998, S. 81 - 92.

J.M. BUCHANAN und G. TULlOCK (1962), The Calculus of Consent: Logical Foundations of Constitutional Democracy, University of Michigan Press, Ann Arbor 1962.

A. BURGESS (2012), 'Nudging' Healthy Lifestyles: The UK Experiments with the Behavioural Alternative to Regulation and the Market, European Journal of Risk Regulation 1/2012, S. 3 - 16.

C.F. CAMERER, S. IssacharofF, G. Loewenstein, T. O’Donoghue und M. RABin (2003), Regulation for Conservatives: Behavioral Economics and the Case for 'Asymmetric Paternalism', University of Pennsylvania Law Review 151 (2003), S. 1211 - 1254.

B. CAPlan (2007), The Myth of the Rational Voter: Why Democracies Choose Bad Policies, Princeton University Press, Princeton N.J. 2007.

B.I. CARLin, S. Gervais und G. MANSO (2011), Libertarian Paternalism and the Production of Knowledge, Mimeo, UCLA, May 2011.

P. CHANDON, J.W. HutChinson, E.T. BradLOW und S.H. Young (2009), Does In-Store Marketing Work? Effects of the Number and Position of Shelf Facing on Brand Attention and Evaluation at the Point of Purchase, Journal of Marketing 73 (2009), S. 1 - 17. 
J.J. Choi, D. Laibson, B. Madrian und A. Metrick (2003), For Better or For Worse: Default Effect and 401(k) Savings Behavior, in: D. WISE (ed.), Perspectives in the Economics of Aging, University of Chicago Press, Chicago 2003, S. 81 - 121.

J.J. ChOi, D. Laibson, B. MAdRian und A. Metrick (2003a), Optimal Defaults, American Economic Review 93 (2003), S. 180 - 185.

R. COASE (1960), The Problem of Social Cost, Journal of Law and Economics 3 (1960), S. 1 - 44; deutsche Übersetzung: Das Problem der sozialen Kosten, in: H.-D. AssmanN, C. KIRCHNER und E. SCHANZE (eds.) (1978), Ökonomische Analyse des Rechts, Athenäum, Kronberg 1978, S. 146 - 202. (Neuauflage: Mohr (Siebeck), Tübingen 1993.).

CH. Cordes und CH. Schubert (2011), Role Models that Make You Unhappy: Light Paternalism, Social Learning and Welfare, Max Planck institute of Economics, Papers on Economics and Evolution, Jena, Januar 2011.

D. Cutler, A. Deaton und J.M. Shapiro (2003), Why Have Americans Become More Obese, Journal of Economic Perspectives 13.3 (2003), S. 93 - 118.

N. DANIELS (ed.) (1975), Reading Rawls: Critical Studies on Rawls 'Theory of Justice', Blackwell, Oxford 1975.

H. DiXon, M. Scully und K. PARKINSON (2006), Pester power: Snackfoods Displayed at Supermarket Checkouts in Melbourne, Australia, Health Promotion Journal of Australia 17 (2006), S. 124 127.

J.S. Downs, G. Loewenstein und J. WisDOM (2009), Strategies for Promoting Healthier Food Choices, American Economic Review (Papers and Proceedings) 99.2 (2009), S. 159 - 164.

X. DrÈzE, ST.J. Hoch und M.E. PURK (1994), Shelf Management and Space Elasticity, Journal of Retailing 70 (1994), S. 301 - 326.

G. DWORKIN (2005), Moral Paternalism, Law and Philosophy 24 (2005), S. 305 - 319.

J. Elster (1979), Ulysses and the Sirens, Studies in Rationality and Irrationality, Cambridge University Press, Cambridge 1979.

J. Elster (2000), Ulysses Unbound, Cambridge University Press, Cambridge 2000.

EUROTRANSPLANT INTERNATIONAL FOUNDATION (2011), Annual Report 2011, Leiden, 2011.

J. FeInBERg (1986), The Moral Limits of the Criminal Law Volume 3: Harm to Self, Oxford University Press, Oxford 1986.

H.G. FRANKFuRT (1971), Freedom of the Will and the Concept of a Person, Journal of Philosophy 68 (1971), S. $5-20$.

B.S. Frey und W.W. Pommerehne (1990), Public Promotion of the Arts: A Survey of Means, Journal of Cultural Economics 14 (1990), S. 73 - 95.

M.M. GALizi (2012), Label, Nudge or Tax? A Review of Health Policies for Risky Behaviours, Journal of Public Health Research 1:e5 (2012), S. 14 - 21.

E.L. GlAeser (2006), Paternalism and Psychology, University of Chicago Law Review 73 (2006), S. 133-156.

W. Glod (2008), How Not to Argue Against Paternalism, Reason Papers 30 (2008), S. 7 - 22.

J. GRUBer Und B. Koszegi (2004), Tax Incidence When Individuals are Time-Inconsistent: The Case of Cigarette Excise Taxation, Journal of Public Economics 88 (2004), S. 1959 - 1987.

T. GRÜNE-YANOFF (2012), Old Wine in New Casks: Libertarian Paternalism Still Violates Liberal Principle, erscheint in: Social Choice and Welfare.

F.A. V. HAYEK (1945), The Use of Knowledge in Society, American Economic Review 35 (1945), S. $519-530$.

F.A. V. HAYEK (1960), The Constitution of Liberty, Routledge and Kegan Paul, London 1960; deutsche Übersetzung: Die Verfassung der Freiheit, Mohr (Siebeck), Tübingen 1971. 
F.A. V. HAYeK (1977), Drei Vorlesungen über Demokratie, Gerechtigkeit und Sozialismus, Mohr (Siebeck), Tübingen 1977.

J.G. HEAD (1966), On Merit Goods, Finanzarchiv NF. 25 (1966), S. 1 - 29.

CH. Jolls und C.R. SunSTEIN (2006), Debiasing Through Law, Journal of Legal Studies 35 (2006), S. $199-241$.

D.R. JUST und C.R. PAYNE (2009), Obesity: Can Behavioral Economics Help?, Annals of Behavioral Medicine 38 (Supplement) (2009), S. 47 - 55.

G. KIRCHGÄSSNER (1992), Towards a Theory of Low-Cost Decisions, European Journal of Political Economy 8 (1992), S. 305 - 320.

G. KIRCHGÄSSNER (1994), Constitutional Economics and Its Relevance for the Evolution of Rules, Kyklos 47 (1994), S. 321 - 339.

G. KIRCHGÄSSNER (2008), Homo Oeconomicus, Das ökonomische Modell individuellen Verhaltens und seine Anwendung in den Wirtschafts- und Sozialwissenschaften, J.B.C. Mohr (Paul Siebeck), 3. Auflage 2008.

G. KIRCHGÄSSNER (2010), On Minimal Morals, European Journal of Political Economy 26 (2010), S. $330-339$.

H. KLIEMT (1986), The Veil of Insignificance, European Journal of Political Economy 2 (1986), S. $333-344$.

F. KосH (2000), Der Aufbruch der Pädagogik, Europäische Verlagsanstalt, Hamburg 2000.

P. Korman und H. Prast (2010), What Does Behavioral Economics Mean for Policy? Challenges for Savings and Health Policies in the Netherlands, De Economist 158 (2010), S. 101 122.

D. Laibson, A. Repetto und J.B. Tobacman (2007), Estimating Discount Functions with Consumption over the Lifecycle, NBER Working Paper Nr. 13314, August 2007.

Th.C. LeOnARD (2008), Besprechung von R.H. Thaler und C.R. Sunstein (2008), Constitutional Political Economy 19 (2008), S. 356 - 360.

G. LOEWENSTEIN und E. HAISLEY (2008), The Economist as Therapist: Methodological Ramifications of 'Light Paternalism', in: A. CAPLIN und A. SCHOTTER (eds.), The Foundations of Positive and Normative Economics, Oxford University Press, Oxford et al. 2008, S. 210 - 245.

G. Loewenstein, T. BrennAn und K.G. VolpP (2007), Asymmetric Paternalism to Improve Health Behaviors, Journal of the American Medical Association 298 (2007), S. 2415 - 2417.

G. LUCAS (2012), Saving Smokers from Themselves: The Paternalistic Use of Cigarette Taxes, University of Cincinnati Law Review 80 (2012), S. 1 - 55.

B.C. MADRIAN UND D.F. SHEA (2001), The Power of Suggestion: Inertia in 401(k) Participation and Savings Behavior, Quarterly Journal of Economics 66 (2001), S. 1149 - 1187.

P. D. MARneffe (2006), Avoiding Paternalism, Philosophy and Public Affairs 34 (2006), S. 68 94.

J.S. MiLL (1859), On Liberty, J.W. Parker, London 1859; zitiert nach der deutschen Übersetzung: Die Freiheit, Wissenschaftliche Buchgemeinschaft, Darmstadt 1967.

A. MoRs (1986), Die Entwicklung der Schulpflicht in Deutschland, Dissertation, Universität Tübingen, 1986.

R.A. Musgrave (1957), A Multiple Theory of Budget Determination, Finanzarchiv NF 24 (1957), S. $333-343$.

R. NOzICK (1974), Anarchy, State, and Utopia, Basil Blackwell, Oxford 1974.

T. O’Donoghue Und M. RABin (2003), Studying Optimal Paternalism, Illustrated by a Model of Sin Taxes, American Economic Review (Papers and Proceedings) 93.2 (2003), S. 186 - 191. 
T. O’Donoghue Und M. Rabin (2006), Optimal Sin Taxes, Journal of Public Economics 90 (2006), S. $1825-1849$.

W.W. POMMEREHNE (1975), Budgetäre Umverteilung in der Demokratie: Ein empirischer Test alternativer Hypothesen, Zeitschrift für Wirtschafts- und Sozialwissenschaften 95 (1975), S. 327 - 364.

W.W. POMMEREHNE und F. SCHNEIDER (1985), Politisch-ökonomische Überprüfung des Kaufkraftinzidenzkonzepts: Eine Analyse der AHV-Abstimmungen von 1972 und 1978, in: E.A. BRUGGER und R.L. FREY (eds.), Sektoralpolitik versus Regionalpolitik, Rüegger, Gruesch 1985, S. $75-100$.

TH.M. Pope (2004), Counting the Dragon's Teeth and Claws: The Definition of Hard Paternalism, Georgia State University Law Review 20 (2004), S. 659 - 722.

TH.M. POPE (2005), Is Public Health Paternalism Really Never Justified?, Oklahoma City University Law Review 30 (2005), S. 121 - 207.

TH.M. POPE (2005a), Monstrous Impersonation: A Critique of Consent-Based Justifications for Hard Paternalism, UMKC Law Review 73 (2005), S. 681 - 713.

D. DE Pury, H. HAuser und B. SCHMid (1995), Mut zum Aufbruch: Eine wirtschaftspolitische Agenda für die Schweiz, Orell Füssli, Zürich 1995.

E. QUinet (2004), A Meta-Analysis of Western European External Costs Estimates, Transportation Research D 9 (2004), S. 465 - 476.

J. RAWls (1971), A Theory of Justice, Harvard University Press, Cambridge (Mass.) 1971; deutsche Übersetzung: Eine Theorie der Gerechtigkeit, Suhrkamp, Frankfurt 1975.

A. Rhitalia, C. MCDAvid, S. SueKarRan, L. Myers und A. SnOwdOn (2009), Impact of Presumed Consent for Organ Donation on Donation Rates: A Systematic Review, British Medical Journal 338a3162 (2009) (Online first).

M.J. Rizzo und G. Whiteman (2009), The Knowledge Problem of New Paternalism, Brigham Young University Law Review 2009, S. 905 - 968.

M.J. Rizzo und G. Whiteman (2009a), Little Brother is Watching You: New Paternalism on the Slippery Slope, Arizona Law Review 51 (2009), S. 685 - 739.

S.C. SACHA (2007), Father Knows Best: A Critique of Joel Feinberg's Soft Paternalism, Georgia State University, Philosophy Thesis 17 (2007), http://digitalarchive.gsu.edu/cgi/viewcontent.cgi?article=1016\&context=philosophy_theses.

W. SAmuelson und R. ZeckHAuser (1988), Status Quo Bias in Decision Making, Journal of Risk and Uncertainty 1 (1988), S. 7-59.

T.C. SCHELLiNG (1978), Egonomics, or the Art of Self-Management, American Economic Review, Papers and Proceedings 68.2 (1978), S. 290 - 294.

T.C. Schelling (1980), The Intimate Contest for Self-Command, Public Interest 60 (1980), S. 94 $-118$.

K. SCHMIDT (1988), Mehr zur Meritorik: Kritisches und Alternatives zu der Lehre von den öffentlichen Gütern, Zeitschrift für Wirtschaft- und Sozialwissenschaften 108 (1988), S. 383 - 403.

J. SCHNELLENBACH (2011), Wohlwollendes Anschubsen: Was ist mit liberalem Paternalismus zu erreichen und was sind seine Nebenwirkungen?, Perspektiven der Wirtschaftspolitik 12 (2011). S. $445-459$.

J. SCHNELlenbach (2012), Nudges and Norms: On the Political Economy of Soft Paternalism, European Journal of Political Economy 28 (2012). S. 266 - 277.

E. Seliger und K.P. Whyte (2012), Nudging Cannot Solve Complex Policy Problems, European Journal of Risk Regulation 1/2012, S. 26 - 31.

A.K. SEN (1977), Rational Fools: A Critique of the Behavioural Foundations of Economic Theory, Philosophy and Public Affairs 6 (1976/77), S. 317 - 344. 
R. SHAFER-LANDAU (2005), Liberalism and Paternalism, Legal Theory 11 (2005), S. 169 - 191.

V. STOCKÉ (2002), Framing und Rationalität: Die Bedeutung der Informationsdarstellung für das Entscheidungsverhalten, Oldenbourg, München 2002.

R. Sugden (2008), Why Incoherent Preferences Do Not Justify Paternalism, Constitutional Political Economy 19 (2008), S. 226 - 248.

C.R. SUnSTEIN und R.H. THALER (2003), Libertarian Paternalism Is Not an Oxymoron, University of Chicago Law Review 70 (2003), S. 1159 - 1202.

R.H. Thaler (1992), The Winner's Curse, Paradoxes and Anomalies of Economic Life, Princeton University Press, Princeton N.J. 1992.

R.H. ThaleR Und S. BenARTZI (2004), Save More Tomorrow: Using Behavioral Economics to Increase Employee Saving, Journal of Political Economy 112 (2004), S. S164 - S187.

R.H. ThaleR und C.R. Sunstein (2003), Libertarian Paternalism, American Economic Review (Papers and Proceedings) 93.2 (2003), S. $175-179$.

R.H. Thaler und H.M. SHEFRIN (1981), An Economic Theory of Self-Control, Journal of Political Economy 89 (1981), S. 392 - 406.

R.H. ThAlER und C.R. SunsteIN (2008), Nudge: Improving Decisions about Health, Wealth, and Happiness, Yale University Press, New Haven, CT, 2008; deutsche Übersetzung: Nudge: Wie man kluge Entscheidungen anstösst, Econ, Berlin 2009.

M. TIETZEL und CH. MÜLleR (1998), Noch mehr zur Meritorik, Zeitschrift für Wirtschafts- und Sozialwissenschaften 118 (1998), S. 87 - 127.

A. TVERSKY und D. Kahneman (1981), The Framing of Decisions and the Psychology of Choice, Science 211 (1981), S. 453 - 458.

A. TVERSKY und D. KAHNEMAN (1987), Rational Choice and the Framing of Decisions, in: R.M. Hogarth und M.W. Reder (eds.), Rational Choice: The Contrast Between Economics and Psychology, Chicago University Press, Chicago 1987, S. 67 - 94.

K. WICKSELL (1896), Finanztheoretische Untersuchungen, Gustav Fischer, Jena 1896.

J. WISDOM, J.S. DOWNS und G. LOEWENSTEIN (2010), Promoting Health Choice: Information versus Convenience, American Economic Journal: Applied Economics 2 (2010), S. 164 - 178.

G. Whiteman (2006), Against the New Paternalism: Internalities and the Economics of SelfControl, Policy Analysis Nr. 563, Cato Institute, 22. Februar 2006.

G. Whiteman und M.J. Rizzo (2007), Paternalist Slopes, New York University Journal of Law and Liberty 2 (2007), S. $411-443$.

G.A. Withers (1979), Private Demand for Public Subsidies: An Econometric Study of Cultural Support in Australia, Journal of Cultural Economics 3 (1979), S. 53 - 61. 